\title{
EXTRA ORDINEM. BREVE STORIA DEL COSTITUZIONALISMO MULTILIVELLO TRA VOLONTÀ D'INTEGRAZIONE, DIALETTICA DELLE DIFFERENZE, GLOBALIZZAZIONE E TRASFORMAZIONI DELLO STATO
}

\author{
Rosamaria AliBRANDi \\ Dottore di Ricerca \\ Storia delle Istituzioni Politiche \\ e Giuridiche dell'Età Medievale e Moderna \\ Università degli Studi di Messina \\ ralibrandi@unime.it
}

\begin{abstract}
RESUMEN
El equilibrio entre la soberanía nacional de cada Estado y la europea ha llegado a un punto crítico. El conocimiento del proceso constituyente de la Unión Europea $y$ de su sistema de governance implica el análisis de la fase de transición que las asambleas representativas están atravesando y que pone en tela de juicio su papel institucional. La globalización, además, ha cambiado de modo irreversible las relaciones interpersonales en el contexto político, económico, territorial y, sobre todo, jurídico. El derecho, instrumento primario de regulación y mediación de las instancias individuales y colectivas y vehiculo para la materialización de las necesidades de una sociedad, debe tener en cuenta la red mundial de conexiones espaciales y las interdependencias funcionales siempre más importantes. La dimensión europea desquicia los confines puestos por las constituciones nacionales; el cambio, más allá de las evidentes recaídas en el Derecho privado, reviste decisivamente una dimensión pública. Los intereses se intersecan pues en un terreno constitucional a varios niveles: el modelo de governance europea responde a criterios de legitimación democrática y de control distintos de los nacionales; el desplazamiento del nivel normativo implica consecuencias para el sistema y los equilibrios constitucionales internos. El proceso constitucional europeo debería caracterizarse como experimentación global de una nueva governance poliárquica, directamente deliberativa, capaz de regenerar los circuitos democráticos.
\end{abstract}

Palabras clave: constitucionalismo, gobierno, integración, Derecho común, Unión Europea, multinivel.

\section{ABSTRACT}

Balance between each state's sovereignty and the European one has reached its peak point. Knowledge of the UE's constituent process as well as its system of government implies the study of the transition stage which representative assemblies 
are going through and which questions its institutional role. Besides, globalisation has clearly changed interpersonal relationships from a political, economic, territorial and legal point of view. The law, a basic tool to rule and mediate among individuals or institutions, as well as a means to put into practice a society's needs should take into account the key role of the global network of space connections and functional interchanges. Its European aspect drives the boundaries set by the countries' constitutions to despair; a change - far beyond the clear relapses which take place in private law - conclusively disguises a public aspect. Then different interests collide in a constitutional land on different levels: the model of European government implies a democratic legitimacy of control different from the one countries have; the transfer of norms implies some consequences for the system and the internal constitutional balance. European constitutional proceedings should be made of a global experience leading to polygarchy, which is a type of government directly deliberative and which can renew democratic circuits.

Keywords: Constitutionalism, government, integration, Common Law, EU, multilevel.

\section{ZUSAMMENFASSUNG}

Das Gleichgewicht zwischen der nationalen Souveränität jedes einzelnen Staates und der Souveränität der EU hat einen kritischen Punkt erreicht. Die Kenntnis der Verfassung der EU und ihres Governance-Systems impliziert die Analyse des Übergangs, welchen die repräsentativen Versammlungen derzeit durchlaufen, welche Ibre institutionelle Funktion in Frage stellt. Die Globalisierung bat zudem, in unumkebrbarer Weise, die interpersonalen Beziebungen im politischen, wirtschaftlichen, territorialen und vor allem im juristischen Kontext verändert. Das Recht, als primärem Instrument der Regelung und Vermittlung zwischen individuellen und kollektiven Instanzen und als Mittel zur Materialisierung der Bedürfnisse einer Gesellschaft, sollte stets das weltweite Netz der territorialen Verbindungen und die wichtigsten funktionalen Abhängigkeiten untereinander berücksichtigen. Die europäische Dimension verunsichert die Abgrenzungen, welche sich die nationalen Verfassungen gegeben haben. Die Veränderungen, die sich jenseits der Rückfälle im Privatrecht zeigten, verweisen entschieden auf eine öffentlich rechtliche Dimension. Die Interessen überschneiden sich verfassungsrechtlich auf verschiedenen Ebenen: das europäische Modell des Governance entspricht den Kriterien der demokratischen Legitimation und einer Steuerung, die jenseits der nationalen Kriterien liegt. Die Verlagerung des normativen Niveaus impliziert Konsequenzen für das System und das interne Gleichgewicht der nationalen Verfassungen. Der europäische konstitutionelle Prozess sollte charakterisiert werden als globale Erfahrung einer neuen polyarchischen governance, die direkt beabsichtigt und fähig ist, die demokratischen Schaltkreise zu regenerieren.

Schlüsselwörter: Konstitutionalismus, Regierung, Integration, Gemeines Recht, Europäische Union, Multilevel.

SUMARIO: I. VERSO LA MULTILEVEL GOVERNANCE.-II. L'INTEGRAZIONE GIURIDICA EUROPEA E L'INELUDIBILE RELAZIONE FRA STORIA E DIRITTO.-III. LA FORTUNA E LE SFIDE DEL MULTILEVEL COSTITUTIONALISM.-IV. LA VICENDA STORICA DEL MOVIMENTO COSTI- 
TUZIONALISTICO EUROPEO.-V. PRIMA CHE SIA TROPPO TARDI. IL RECUPERO DEL SIGNIFICATO STORICO DELLA VOCE «COSTITUZIONE».-VI. IL COSTITUZIONALISMO MULTILIVELLO NELL'IPOTESI DI UNA COSTITUZIONE EUROPEA.-VII. UNIONE POLITICA O COSTRUZIONE GIURIDICA? LE POSSIBILI CONCLUSIONI.

«Quivi tue nozze si faranno, e tosto

Da me tu figli avrai, famosi figli, Cui scettro si darà sul mondo intero. Disse, e al suo favellar fu pari il fatto. Apparve Creta; e Giove altra sembianza vestì; disciolse alla donzella il cinto:

L' Ore acconciaro il talamo, ed Europa Che vergine era ancor, del sommo Giove Divenne sposa, concepì, fu madre».

(Giacomo LeOPARDI, Europa)

\section{VERSO LA MULTILEVEL GOVERNANCE}

$\mathrm{Ci}$ inoltriamo in un'era nella quale antiche parole-chiave, emblemi di nette connotazioni e appartenenze, trasmutano da un'area epistemologica a un'altra, deprivate dell'originario significato, per essere riutilizzate in una dimensione astorica e acritica in nome del mito della fine delle ideologie, e persino della fine della storia ${ }^{1}$.

Il recupero dello spazio di un pensiero critico in grado di ritornare all'autentica radice semantica e ideologica delle parole della politica, forando la placenta della sua intrinseca retorica, comporta una interpreta-

${ }^{1}$ La teorizzazione della fine della storia (F. FunuYAma, The End of History and the Last Man, New York, The Free Press, 1992; traduzione italiana D. Ceni, La fine della storia e l'ultimo nomo, Milano, BUR, 2003), pur se facilmente criticabile, in quanto la storia non finisce nel momento in cui si esaurisce un determinato modello, postula che, con la caduta dell'Unione Sovietica, abbiamo avuto la fine della contrapposizione tra due diversi modelli di società e di economia con la vittoria di uno di essi. Questo avrebbe chiuso la vicenda della dialettica storica: non c'è più una storia alternativa ma c'è solo una storia all'interno di quel determinato modello che è risultato vincente. Sia consentito il rinvio, sul punto, a R. Alibrandi, Rivoluzione Sovranità Libertà. L'aurora della modernità, Napoli, La Città del Sole, 2016, pp. 236-237, n. 19. Sull'attuale svalutazione, in generale, delle regole democratiche e, in particolare, sulla crisi della forma rappresentativa e della dimensione costituzionale della democrazia, che si esprime nel rifiuto del paradigma dello Stato costituzionale di diritto quale sistema di vincoli legali imposti a qualunque potere, si veda L. FerRAJOLI, Poteri selvaggi. La crisi della democrazia italiana, Roma-Bari, Laterza, 2012. 
zione del presente nel contempo filologica e innovativa, che si snodi lungo un lessico di voci, tanto antiche da sembrare perenni, quali repubblica, costituzione, democrazia, eguaglianza, maggioranza, opposizione, governabilità, legalità, e altre ancora, per non tacere di alcune d'uso più corrente quali lavoro o povertà, o, tout court, futuro, il cui esame critico serva a comprendere l'enormità della posta in gioco d'una partita epocale.

Superato il livello nazionale, questa teoria di lemmi va declinata nell'area prospettica dell'Unione Europea, ove si staglia un'architettura complessa e multilivello davvero peculiare. Come è noto, è obiettivo dell'Unione la creazione di un'entità europea governabile, efficiente e democratica. E' quindi auspicabile che la forma di governance, a più livelli, nella quale organizzazioni politiche sovranazionali assumono competenze tradizionalmente riservate allo Stato nazionale, si articoli secondo una pluralità di poteri limitati, che si controllino reciprocamente, alla cui guida vi sia alternanza, e sull'esercizio dei quali il giudizio venga rimesso ai cittadini. Occorre, sostanzialmente, che la logica che sottende a una siffatta poliarchia si fondi sull'equilibrio.

Una poliarchia europea ${ }^{2}$, quale nuova architettura politica che veicoli un processo di democratizzazione, volto a evitare che il sistema si evolva verso la costituzione di una oligarchia che prenda decisioni per la massa dei cittadini, realizza i suoi fini solo se essa stessa assuma una forma democratica e leghi i suoi processi decisionali alla più ampia discussione possibile riguardo a ogni decisione di rilevanza pubblica. Si configura pertanto come una poliarchia direttamente deliberativa qualora vi sia la diretta partecipazione dei cittadini alla soluzione dei problemi che li riguardano; se le deliberazioni che investono il pubblico siano giustificate, e, infine, quando il potere, articolato in modo poliarchico, circoli nella società e non sia centralizzato in nessun organo politico specifico ${ }^{3}$. Appare perspicuo che i per-

${ }^{2}$ Già nel 1956, in un magistrale saggio sulla dottrina, e sui fini, della democrazia — scritto in un'era ormai conclusa da tempo, nella quale il «modello alternativo» era costituito dal blocco sovietico-, Robert Dahl trattava di polyarchal democracy, definendo le norme e le condizioni perché una polyarchy potesse esistere, essere usata e funzionare. R. DAHL, A Preface to Democratic Theory, cap. 3, Polyarchal Democracy, Chicago, University of Chicago Press, 1956, pp. 63 e ss., in particolare pp. 80-86.

3 J. CoHEN e C. F. SABEL, «Directly-Deliberative Polyarchy», European Law Journal, vol. 3 , n. 4 (1997), pp. 313-340. Al di là di una visione meramente politico-giuridica, interessanti argomentazioni sugli effetti della polyarchic governance nel mondo del lavoro, sia nel settore privato che, in modo particolare, in quello pubblico, dimostrano come essa sia funzionale, per manipolare i professionals evitando ogni scontro diretto, a una central oligarchy, che «within polyarchic modes of governance [...] unobtrusively retains strategic control over middle and lower level elites by opening up the herarchically containable horizontal exercise of delegated leadership», mettendo in atto una sorta di informal mechanism of co-optation orizzontale 
corsi non siano semplici, e che i processi di governance realizzati in forza del principio poliarchico sembrino sperimentali più che concreti, considerato che generano dubbi sulla loro stessa assunzione e pratica, e producono, per ottimizzare gli scopi, un continuo «readjustement of ends and means through comparison of different approaches to advancing common general aims» ${ }^{4}$. La soluzione dei problemi non è un miracolo che scaturisce da una presupposta armonia, inesistente nella realtà; dipende, piuttosto, da un continuo squilibrio, dall'allineamento imperfetto di interessi sia convergenti che confliggenti, dall'esplorazione delle differenze. Ecco perché molte deliberazioni concrete dell'UE possono essere definite poliarchiche ${ }^{5}$. Il processo decisionale che a esse sottende, subordina l'esercizio del potere collettivo alla disciplina della ragione, alla forza dell'argomento migliore ${ }^{6}$, razionalmente motivante anche quando non coincida con l'interesse di chi occupa una posizione di supremazia.

Per l'Italia, come per gli altri Stati membri, ogni programma di riforme non è realizzabile se non correlato alle linee guida europee. Ma l'interrelazione tra la sovranità dei singoli Stati e quella europea è giunta a un punto critico. Come va concepito il governo politico dell'Europa? Come va perseguito il fine della sua piena legittimazione? Il parlamento europeo eletto direttamente dai cittadini resta un'essenziale conquista democratica; occorre tuttavia incentivare una governance «multilivello» che tenga conto di competenze e sovranità distinte, oltre che di quelle condivise ${ }^{7}$.

La conoscenza del processo costituente dell'Unione Europea e del suo sistema di multilevel governance 8 implica, a priori, l'analisi della difficile fase di transizione che le assemblee rappresentative stanno attraversando,

senza inficiale la scala gerarchica del potere. Accade quindi che, lungi dall'implementare sistemi democratici, forme ibride, e tuttavia istituzionali, generate dalla poliarchia, attribuiscano un ruolo dominante «to leadership to all levels of the hierarchical authority structures through which elite rule is reproduced and legitimated». M. L. REED, «Leadership and "Leaderism". The Discourse of Professional Leadership and the Practice of Management Control in Public Services», in M. Dent, I. L. Bourgeault, J.-L. Denis e E. Kuhlmann (curr.), The Routledge Companion to the Professions and Professionalism, Abingdon, Routledge, 2016, pp. 200-216.

${ }^{4}$ C. F. Sabel e J. Zeitlin, «Experimentalist Governance», in D. Levi-Faur, The Oxford Handbook of Governance, Oxford, Oxford University Press, 2012, pp. 169-187, in particolare p. 170.

5 J. Cohen e C. F. SABel, «Directly-Deliberative Polyarchy», op. cit., p. 326.

${ }^{6} \mathrm{~J}$. Habermas, Agire comunicativo e logica delle scienze sociali, Bologna, Il Mulino, 1980, p. 321.

7 A. Giacomini e G. Costanzo, Europa al plurale. Lettera alla professoressa ungherese Teodora, Roma, Armando Editore, 2015, p. 33.

${ }^{8}$ P. Bilancia, The Dynamics of the EU Integration and the Impact on the National Constitutional Law, Milano, Giuffrè, 2012, pp. 1-12. 
che rimette in discussione il loro ruolo istituzionale. Negli ultimi decenni, il processo di integrazione europea e, a livello d'ogni singola nazione, la ricerca di garanzie di governabilità e di efficienza dell'azione politica, hanno progressivamente spostato il baricentro della decisione dai parlamenti ai governi. Se il parlamento, per tradizione, rappresenta il luogo della ricerca del compromesso politico, i governi sono la sede della concreta definizione e attuazione degli indirizzi programmatici, funzione che rileva, in particolar modo, nel corso d'una recessione economica.

Si è venuta configurando una realtà nuova; la globalizzazione ha cambiato in modo irreversibile le relazioni interpersonali in ambiti territoriali, economici, sociali e giuridici, a loro volta mutati. Anche il diritto, difatti, quale strumento primario di regolazione e di mediazione delle istanze individuali e collettive, veicolo per dar forma ai bisogni di una società, deve tener conto della rete mondiale di connessioni spaziali e di interdipendenze funzionali. Dallo scenario mondiale, affollato da modalità di estrinsecazione del diritto che superano o eludono i confini statali, emerge un assetto mobile, nell'ambito del quale si son venute configurando classi giuridiche inedite - spazio giuridico globale, globalismo giuridico, internazionalismo giudiziario - a riprova dell'esistenza d'una vasta gamma di nuovi soggetti e oggetti del diritto, come pure della molteplicità delle nuove sue fonti, sia nazionali che internazionali 9 .

Le categorie tradizionali del diritto interno, così come quelle del diritto internazionale, appaiono, dunque, inadatte alla riflessione sui fenomeni emergenti. Lo Stato nazionale, come concetto prima che come realizzazione, attraversa una crisi dalla quale deriva la necessità di rivedere i meccanismi e le forme della legittimazione democratica e, dall'altra, la modificazione del legame esclusivo tra Stato e cittadini a favore di una pluralità di appartenenze, di identità e quindi di diritti. Ai processi di stratificazione dei poteri, da quelli internazionali e sopranazionali a quelli infrastatali, fanno riscontro crescenti differenziazioni del regime che regola i diritti, con l'affermazione di diversi livelli di tutela che segnano sempre più il superamento del dogma del costituzionalismo classico del monopolio statale in materia.

L'interazione tra livelli di tutela e ordinamenti giuridici e valoriali di appartenenza del singolo ha generato sistemi a più livelli di protezione e

9 Per una approfondita analisi del concetto di globalismo giuridico, si veda D. ZoLO, Tramonto globale. La fame, il patibolo, la guerra, Firenze, Firenze University Press, 2010, pp. 54-62, e, sul processo di globalizzazione del diritto, i saggi di M. R. FERRARESE, Diritto sconfinato: Inventiva giuridica e spazi nel mondo globale, Roma-Bari, Laterza, 2015, e ID., Prima lezione di diritto globale, Roma-Bari, Laterza, 2012. 
salvaguardia dei diritti dell'uomo ${ }^{10}$. I diritti nascono con lo Stato «fino al punto di giustificare la scelta della stessa organizzazione statale»; ma ora «da un modello, quale quello ottocentesco nel quale lo Stato viene dopo i diritti, si è passati ad un modello nel quale lo Stato e diritti nascono insieme, i secondi legittimando il primo» ${ }^{11}$.

Nell'attuale agone politico e normativo non sono del tutto definite le delimitazioni tra lo spazio giuridico ancora riservato alla legislazione e alla giurisdizione interna e lo spazio giuridico europeo ${ }^{12}$. Le costituzioni democratiche contemporanee individuano nei diritti lo spazio di affermazione e sviluppo della personalità e il principio di organizzazione della società. In tal modo i diritti rappresentano i bisogni individuali e di gruppo e rivelano i valori la cui tutela lo Stato assume come obiettivo della sua azione e come limite della propria sovranità ${ }^{13}$.

Un tema di così pressante attualità politica e di tale interesse giuridico, come quello delle trasformazioni dello Stato costituzionale in Europa, studiato dalla prospettiva eurounitaria - con particolare attenzione al caso italiano- per interpretare le tensioni che investono l'ordine costituzionale e provano a ridisegnare i confini delle diverse costituzioni nazionali, alterandone la natura originaria, induce a riflettere su come vada articolato il potere esecutivo in un contesto in cui la frantumazione del territorio, il complesso sistema dei mercati e la globalizzazione impongono la necessità di ripensare criticamente $\mathrm{i}$ tradizionali dogmi del costituzionalismo ${ }^{14}$.

${ }^{10}$ E. MotTeSE, «Tutela multilivello dei diritti fondamentali e teoria dei contro limiti. Il sistema europeo di protezione dei diritti umani nei rapporti tra corte costituzionale, corte di giustizia e corte europea dei diritti dell'uomo: una integrazione limitata», Fogli di Lavoro per il diritto internazionale, disponibile in http://www.lex.unict.it/crio/foglidilavoro/12010_ contributi.pdf, pp. 1-2.

${ }^{11}$ P. CARETTI, «La tutela dei diritti fondamentali nella prospettiva della Costituzione Europea», in P. BILANCIA e E. De Marco (curr.), La tutela multilivello dei diritti: punti di crisi, problemi aperti, momenti di stabilizzazione, Milano, Giuffrè, 2004, pp. 75 e ss.

${ }_{12}$ G. SILVESTRI, «Verso uno ius commune europeo dei diritti fondamentali», Quaderni Costituzionali, n. 1 (2006), pp. 6-24, in particolare p. 11.

${ }^{13}$ G. Silvestri, «La parabola della sovranità. Ascesa, declino e trasfigurazione di un concetto», Rivista di Diritto Costituzionale, n. 1 (1996), pp. 3-74, ora in G. SILVESTRI, Lo Stato senza principe, La sovranità dei valori nelle democrazie pluraliste, Torino, G. Giappichelli, 2005, pp. 36-43.

${ }^{14}$ Sull'impatto della globalizzazione nella sfera del diritto vi è una vasta letteratura giuridica internazionale. Tra i molti autori, a parte il classico, recentemente ristampato, R. J. HoLTON, Globalization and the Nation-State, Basingstoke, Palgrave, 1998, ricordiamo ID., Making Globalization, Basingstoke, Palgrave, 2005; W. TwInIng, Globalization and Legal Theory, Cambridge, Cambridge University Press, 2000; ID., General Jurisprudence. Understanding Law from a Global Perspective, Cambridge, Cambridge University Press, 2009; A. HalPIN e V. Roeben (curr.), Theorising the Global Legal Order, Oxford, Hart, 2009; B. Sousa SAN- 
La crisi dello Stato nazionale, come avvenne nel passaggio tra Ottocento e Novecento ${ }^{15}$, postula una ridefinizione dei termini del rapporto tra Stato e società ${ }^{16}$. Si evidenzia una subordinazione del pubblico al privato secondo direttrici che in parte rievocano le linee guida dello Stato liberale ottocentesco ${ }^{17}$.

Parte della dottrina prova a dare forma a una nuova governance mondiale che si ponga oltre lo Stato e il pubblico, favorendo i networks e le auto-organizzazioni del privato. Altri studi, riconducibili al neo-costituzionalismo, hanno come prospettiva la formazione di un governo mondiale e la visione del diritto internazionale quale nuova forma di diritto costituzionale. Al centro del dibattito costituzionale rimane la nozione primaria della forma dello Stato, ripensato nell'ambito di una struttura multilivello, per il quale abbiano rilevanza sia gli Enti territoriali che l'Unione Europea. Si delinea uno scenario in cui sarà necessario dotare l'Unione di strumenti che ne colmino il deficit democratico favorendo una sempre maggiore integrazione politica il cui peso «non potrà limitarsi ad una definizione di limiti all'indebitamento o al coordinamento di politiche economiche», ma dovrà estendersi a obiettivi diversi da quelli meramente economici ${ }^{18}$.

TOS, Toward a New Legal Common Sense. Law, Globalization, and Emancipation, Cambridge, Cambridge University Press, 2002; B. Sousa Santos e C. A. Rodríguez-Garavito, Law and Globalization from Below. Towards a Cosmopolitan Legality, Cambridge, Cambridge University Press, 2005; U. La Porta, Globalizzazione e Diritto, Napoli, Liguori, 2006; C. LASCHET, Die Globalisierung in der verfassungstheoretischen Diskussion, München, GRIN, 2007; L. Boulle, The Law of Globalization: An Introduction, Alphen aan den Rijn, Kluwer Law International, 2009; M. FAURE e A. VAN DER WALT, Globalization and Private Law. The Way Forward, Cheltenham, Edward Elgar, 2010; G. Teubner, Verfassungsfragmente: Gesellschaftlicher Konstitutionalismus in der Globalisierung, Berlin, Suhrkamp, 2012; M. R. FERRARESE, Prima lezione di diritto globale, op. cit.; C. JAMIN e W. VAN CAENEGEM, The Internationalisation of Legal Education, Switzerland, Springer, 2016; A. Dignam e M. Galanis, The Globalization of Corporate Governance, London-New York, Routledge, Taylor \& Francis, 2016; S. KIRCHNER, The Status of the Individual in International Law and the Age of Globalization, München, GRIN, 2016, e F. ARcos Ramírez (cur.), La justicia y los derechos en un mundo globalizado, Madrid, Dykinson, 2016.

${ }^{15} \mathrm{Si}$ consenta il rinvio a R. AliBRANDI, «German influence on Italian jurists' perceptions of the British constitutional model in the late nineteenth century», Parliaments, Estates and Representation, n. 2 (2016), pp. 5-6.

${ }^{16}$ E. C. RafFiotTa, Il Governo multilivello dell'economia, Bologna, Bononia University Press, 2013, p. 13.

${ }_{17}$ A. SuPIOT, «The public-private relation in the context of today's refeudalization», International Journal of Constitutional Law, vol. 11, n. 1 (2013), pp. 129-145.

${ }^{18}$ E. C. RafFiotTA, Il Governo multilivello..., op. cit., p. 282. 


\section{L'INTEGRAZIONE GIURIDICA EUROPEA E L'INELUDIBILE RELAZIONE FRA STORIA E DIRITTO}

L'approfondimento della questione dell'integrazione giuridica europea alimenta l'incontro, e spesso lo scontro, fra teorie e ideologie. Da una parte, si colloca la dottrina tradizionale, forte della dogmatica del positivismo giuridico novecentesco. Dall'altra, una scuola giuridica di nuovo tipo, quella dei pratici, dei funzionari delle Comunità europee, dei fautori di tesi federaliste, che influenzano fortemente le forme consuete dell'argomentazione nel campo del diritto pubblico. Emerge l'esigenza di esplicitare i principî sostanziali coinvolti nell'attività giurisdizionale oltre che dottrinale; peraltro, appare chiaro come una Costituzione europea in progress tolleri male ogni cristallizzazione assiomatica. Si trascorre pertanto dalla dogmatica del positivismo giuridico alla retorica del neocostituzionalismo; dalle ricostruzioni dei rapporti fra ordinamenti giuridici sovrani, al bilanciamento fra principî costituzionali. L'integrazione in seno all'Europa mette in discussione le distinzioni fra interno ed esterno all'ordinamento statale, fra giuridico ed extragiuridico, tra ciò che è lecito e legittimo e ciò che, invece, è illecito e illegittimo, e, infine, fra teoria e ideologia ${ }^{19}$.

Se si ipotizza che l'Unione Europea sia un'organizzazione nella quale gli esecutivi centrali dei singoli Stati non governano, ma condividono la responsabilità con altri attori politici, sia a livello sopranazionale che subnazionale, si comprende che, più che una descrizione dello stato di fatto, la multilevel governance sia una teoria, atta a descriverne e interpretarne la natura e il funzionamento. E, tuttavia, sullo sfondo, si addensano incertezze su come l'UE dovrebbe essere organizzata per realizzarla, mentre è intenso il dibattito dottrinale sui limiti posti al programma di integrazione politica europea dalla salvaguardia delle identità costituzionali nazionali, e dall'attuazione della Integrationsverantwortung, ovvero del complesso principio di responsabilità rispetto all'integrazione medesima ${ }^{20}$.

Nello spazio giuridico europeo la governance multilivello emerge essa stessa come principio costituzionale, e prefigura un metodo di governo basato sul forte coinvolgimento degli enti subnazionali nella creazione e attuazione della legislazione e delle politiche dell'Unione Europea.

19 G. Itzcovitch, Teorie e ideologie del diritto comunitario, Torino, Giappichelli, 2016, pp. 19 e 317-319.

${ }^{20}$ C. Panara, The Sub-national Dimension of the EU. A Legal Study of Multilevel Governance, Switzerland, Springer, 2015, pp. 1-2 e 126-127. 
Il suo fondamento risiede nel mosaico derivante dai sistemi costituzionali sia dell'Unione che dei suoi Stati membri. Gli accordi di governance multilivello giocano un ruolo fondamentale nel raggiungimento degli obiettivi chiave del Trattato di Lisbona, quali il rispetto delle identità nazionali, l'autonomia regionale e locale, l'apertura e la chiusura della cittadinanza, tutte disposizioni che conferiscono legittimità e maggiore efficienza al processo decisionale dell'UE, mentre promuovono il costituzionalismo e la democrazia in Europa. Specularmente, il Trattato riflette in molte sue parti l'idea basilare del costituzionalismo multilivello (che contempla il consolidamento di un ordinamento policentrico coerente con una pluralità di testi costituzionali e con i trattati stessi) in particolare nell'individuazione dell'Unione Europea come nuovo sistema istituzionale politico e di governance, caratterizzato da punti fondamentali quali il riconoscimento della posizione centrale dei cittadini, il ruolo dei parlamenti nazionali, il rafforzamento della sovranità europea integrata. Questa sovranità supera l'idea della sovranità statale condivisa con l'UE, ed è più coerente con la dimensione costituzionale del processo di integrazione europea, che contempera il rafforzamento del potere pubblico sovranazionale con le singole costituzioni degli Stati membri, che divengono parti essenziali del sistema multilivello europeo ${ }^{21}$. Il sistema sovranazionale appare vocato ad assicurare una stabilità che gli Stati non sono più in grado di garantire, sia per i processi politici che determinano un'erosione della loro sovranità, che per l'emergere di forme di governance deterritorializzate che mettono in crisi il concetto stesso di sovranità, intesa come supremo potere di comando ${ }^{22}$.

In tale contesto, non si può eludere un interrogativo: qual è oggi il ruolo dei parlamenti nazionali? Una logica politica lungimirante dovrebbe garantire che a governi forti corrispondano parlamenti altrettanto forti; e che le (eventualmente) necessarie modifiche costituzionali richiedano meccanismi che permettano alla macchina democratica di continuare a funzionare. Se lo Stato-nazione non è finito, «il "globale" non impone necessariamente "un nuovo sovrano", piuttosto un differente modo di organizzazione del potere politico» ${ }^{23}$. Peraltro, il richiamo a problemi

${ }^{21}$ Nel 2007 il Trattato di Lisbona, accantonato il progetto del 2004 di una Costituzione europea, ha risposto all'esigenza di riformare la struttura e il funzionamento dell'UE. Si veda G. D'Ignazio, «Le sfide del costituzionalismo multilivello», in G. D'Ignazio (cur.), Multilevel constitutionalism tra integrazione europea e riforme degli ordinamenti decentrati, Milano, Giuffrè, 2011, pp. 13-14.

${ }^{22}$ Sulla sovranità come potere supremo e sul suo paradigma funzionalista, si veda D. Quaglioni, La sovranità, Roma-Bari, Laterza, 2004.

23 E. C. RafFiotTA, Il Governo multilivello..., op. cit., p. 278. 
di equilibrio interno non è peregrino; il concetto di governo multilivello implica, a priori, un bilanciamento dei poteri nazionali, regionali e locali, di politiche territoriali e strategie nazionali in capo a nuovi soggetti con nuovi poteri che affiancano i soggetti istituzionali tradizionali già operativi, e collaudati ${ }^{24}$. Il progressivo inserimento dello Stato nelle varie organizzazioni europee, che si sono susseguite fin dagli anni Cinquanta - mercato comune, assetti comunitari sempre più complessi e, dal 1992, l'Unione sul piano politico e giuridico-, costituisce un evento che Paolo Grossi ha definito enorme per l'esperienza giuridica italiana, e di certo anche per gli altri Stati europei. L'origine del processo di unificazione ha conferito un marchio di tipicità a tutto il suo futuro svolgimento: legata all'esigenza di un mercato comune, di una più efficace cooperazione economica, ha sempre valorizzato nel suo ambito la dimensione economica, che rimane protagonista anche nella costruzione del suo ordinamento giuridico.

Accanto a questa tipizzante attualità, conviene sottolineare un altro profilo dell'Europa giuridica, che rende tipico il suo diritto e costituisce un momento arricchente per ogni singolo Stato sovrano, tessera autonoma del variegato mosaico europeo, che realizza una unità delle diversità

\footnotetext{
${ }^{24}$ Per completare il quadro istituzionale europeo, a proposito di livelli multipli della governance, occorre rilevare la progressiva affermazione delle Autorità Indipendenti, locuzione che indica una serie di poteri pubblici, caratterizzati da uno specifico grado di indipendenza dal potere politico, dall'esercizio di funzioni «neutrali» in diversi settori, specie in quello economico, e da un alto livello di competenze tecniche. Le Autorità Amministrative Indipendenti sono, nel contesto europeo, un'esperienza recente che si è affermata in deroga a due principî cardine degli stati di diritto di matrice liberale: al principio di separazione dei poteri, in quanto le Autorità di solito esercitano poteri che ineriscono non solo alla sfera amministrativa, ma anche a quelle legislativa e giurisdizionale, e al principio su cui si fonda il governo parlamentare, che pone le amministrazioni alle dipendenze dei governi, i quali, a loro volta, rispondono ai parlamenti del proprio operato. Sono nate, in Europa, per realizzare finalità proprie del diritto europeo, quali quelle inerenti al superamento dei monopoli pubblici, ai processi di liberalizzazione e privatizzazione, allo sviluppo di un mercato comune. Si configurano quindi, fin dalla loro prima apparizione nel sistema dell'Unione, come soggetti di natura «bifronte», al confine tra le amministrazioni nazionali e l'amministrazione europea. I problemi che hanno più impegnato le Autorità presenti nell'ambito dell'Unione riguardano, per un aspetto, la loro legittimazione, per un altro, il profilo della loro indipendenza dalla politica, in assenza di specifici supporti costituzionali in grado di giustificare questa nuova presenza. Per quanto concerne il rapporto tra Autorità e istituzioni europee, va rilevato che le direttive europee hanno già definito tale connessione per favorire la nascita di forme di amministrazione integrata, o mista, tra Unione europea e ordinamenti nazionali. Ulteriori interventi potrebbero valorizzare il ruolo del parlamento europeo, poiché, in prospettiva, l'area di competenza delle Autorità Indipendenti di matrice nazionale potrebbe essere l'ambito nel quale sviluppare l'azione di quel sistema parlamentare integrato euronazionale che il Trattato di Lisbona ha inteso avviare. Cfr. sul tema G. P. Cirillo e R. CHIEPPA, Le autorità amministrative indipendenti, Padova, CEDAM, 2010, pp. 3-4.
} 
rispettando le peculiarità storiche delle parti che lo compongono. Il divenire dell'ordinamento giuridico europeo fa sì che l'Unione si identifichi in un itinerario di ricerca, di ricerca di se stessa. Non dimentichiamo, infatti, che, all'inizio e nei suoi primi passi, ci sono solo dei trattati, ossia degli strumenti pattizi fra alcuni Stati. In altre parole, l'Europa non assume una Costituzione o un complesso di leggi quale suo basamento originario. Per questa sua singolarità storica il diritto europeo è tuttora progrediente, e ha quale organo propulsore un organismo giudiziale, la Corte di giustizia (CGUE) con sede in Lussemburgo, più che nel Parlamento o nella Commissione, sua istituzione di governo. L'Europa giuridica si presenta soprattutto come un ordinamento giurisprudenziale, ovvero un ordinamento che trova la sua fonte primaria non in un organismo politico, bensì nell'attività dei giuristi e dei tecnici nell'ambito della loro esperienza professionale ${ }^{25}$; l'operato dei giudici e dei giuristi, in ambito europeo, rileva in modo particolare in quanto è in grado di elaborare le categorie generali ordinanti, necessarie a organismi politico-giuridici dalla straordinaria complessità ${ }^{26}$.

\section{LA FORTUNA E LE SFIDE DEL MULTILEVEL CONSTITUTIONALISM}

Di costituzionalismo multilivello si inizia a parlare all'interno della dottrina tedesca all'indomani del Trattato di Amsterdam ${ }^{27}$ per indicare un sistema in cui l'esercizio dei poteri sovrani da parte del potere pubblico è distribuito su più livelli che trovano in se stessi la propria legittimazione, e sono espressione delle diverse identità politiche dei soggetti interessati ${ }^{28}$.

Storicamente, di sovranità diffusa o ripartita disquisiva già Tocqueville nei primi decenni dell'Ottocento ${ }^{29}$. Questo modello dottrinale, che riecheg-

25 P. Grossi, Il diritto in Italia, oggi, tra modernità e pos-modernità, Lectio brevis, Roma, Accademia Nazionale dei Lincei, 2013, pp. 1-15, in particolare pp. 8-10.

${ }^{26}$ P. Grossi, «Il messaggio giuridico dell'Europa e la sua vitalità: ieri, oggi, domani», Contratto e impresa/Europa, vol. 18, n. 2 (2013), pp. 681 e ss.

${ }^{27}$ Il Trattato venne firmato il 2 ottobre 1997 dagli allora 15 paesi dell'UE, ma entrò in vigore il $1^{\circ}$ maggio 1999 .

${ }^{28}$ In tempi recenti, il concetto è stato analizzato da I. PERNICE, «The Framework revisited constitutional, federal and subsidiarity issues», Colombian Journal of European Law, n. 2 (1996), pp. 403 e ss.

29 A. Tocqueville, La democrazia in America (1835-1840), in G. Candeloro (cur.), Milano, Rizzoli, 2003, p. 67. Sul principio di sovranità nella costruzione del costituzionalismo americano, si consenta il rinvio a R. AlibRANDI, Rivoluzione Sovranità Libertà..., op. cit., pp. $185-188$ e 190-194. 
gia anche le teorie del primo costituzionalismo americano ${ }^{30}$, ha quale obiettivo la costruzione di un'Europa che ha avuto come pilastri dapprima le comunità europee, e la CEE in special modo, e ora l'Unione Europea, che, nello stesso tempo, è l'organizzazione dalla quale la teoria del costituzionalismo multilivello trae origine e a cui è vocata a dare fondamento.

Secondo Ingolf Pernice ${ }^{31}$, al quale si devono la metafora e l'originale teorizzazione del costituzionalismo multilivello, che si colloca nella scuola di pensiero che ritiene attualmente esistente una Costituzione europea quale sistema complesso, la questione Does Europe need a Constitution non sembra rilevante ${ }^{32}$, perchè «Europe already has a "multilevel Constitution": a Constitution made up of the constitutions of the Member States bound together by a complementary constitutional body consisting of the European Treaties». L'Unione Europea è un sistema di potere ripartito in una pluralità di livelli, nella quale «each level of government, regional (or Länder), national (State) and supranational (European), reflects one of two or more possibile identities of citizens concerned. And each of these identities corresponds to a different level of society $»^{33}$. Peraltro, l'illustre costituzionalista, anch'egli tedesco, che aveva posto l'interrogativo, sottolineava, nelle conclusioni dell'acuta analisi d'uno statu quo che non sembra poi così mutato, come il vero accordo legale che unisce due o più nazioni e che come strumento giuridico si attaglia alle esigenze internazionali, è, e rimane, il trattato, in quanto possiede le caratteristiche che consentono il «legal binding of Community power, yet leaves the basic decisions about the Community with Member states, when they can be democratically checked and accounted for. A European constitution would not be able to bridge the existing gap». In definitiva, la legittimazione che una Costituzione europea si trova a mediare sarebbe a fictious one ${ }^{34}$.

La differenza tra le Carte costituzionali dei vari Stati membri e i trattati costitutivi dell'Unione appare dunque, almeno in parte, solo formale; nella

30 A. Hamilton, J. Madison e J. Jay, The Federalist, Cambridge (MA), The John Harvard Library, 2009, pp. 79-83.

${ }^{31}$ Una recentissima, acuta ed esaustiva analisi della teorizzazione di Ingolf Pernice, inclusa la disamina delle critiche dei punti oscuri del multilevel constitutionalism si deve a G. MARTinico, «La complessità costituzionale dell'ordinamento europeo», in A. RUGGERI (cur.), Scritti in onore di Gaetano Silvestri, t. II, Torino, Giappichelli, 2016, pp. 1364-1380, in particolare alle pp. 1364-1370.

32 D. Grimm, «Does Europe Need a Constitution?», European Law Journal, vol. 1, n. 3 (1995), pp. 282-302.

33 I. PERnICE, «Multilevel Constitutionalism and the Treaty of Amsterdam. European Constitution-Making Revisited?», Common Market Law Review, n. 36 (1999), pp. 703-750, in particolare p. 707.

${ }^{34}$ D. GrIMm, «Does Europe Need a Constitution?», op. cit., p. 299. 
sostanza si sarebbe da tempo in presenza di un coerente e unitario sistema istituzionale basato sulla ripartizione tra i diversi livelli di quei poteri che normalmente vengono ricondotti al principio di sovranità.

Il progressivo trasferimento di poteri dal livello statale a quello sopranazionale e substatale mostra la connessione tra la teoria del costituzionalismo multilivello e quelle che riguardano, più in generale, i processi di redistribuzione di quei poteri che tradizionalmente erano prerogativa dello Stato ${ }^{35}$. Lo Stato nazionale tendenzialmente non riconosce un potere superiore, e rivendica il monopolio della forza legittima e l'originarietà del proprio ordinamento quale condizione di efficacia e vigenza del diritto, secondo quel canone disegnato a partire dal XV secolo, affermatosi pienamente nel Seicento, e giunto all'apice nell'Ottocento. Oggi, però, la crescente articolazione del potere su una pluralità di livelli genera una sorta di malfunzionamento dell'ente statale, come se fosse ormai inadeguato a svolgere i compiti storicamente attribuitigli. Quindi, da soggetto, è divenuto oggetto di un processo di erosione delle sue tradizionali prerogative ${ }^{36}$. Un tale sgretolamento di poteri ${ }^{37}$ si sta verificando sia a livello inferiore, al suo interno, che a livello superiore, ed è riconducibile alla globalizzazione, ovvero a una progressiva integrazione, soprattutto di natura economica, delle diverse aree del mondo, determinata dall'inarrestabi-

35 Nella vasta bibliografia sulla crisi dello Stato nazione e sul concetto di sovranità si collocano i classici G. GUARINO, «Pubblico e privato nella economia. La sovranità tra Costituzione e istituzioni comunitarie», Quaderni costituzionali, n. 1 (1992), pp. 21 e ss.; M. LuCIANI, «Sovranità e diritti fondamentali», Critica marxista, n. 5 (1993), pp. 20 e ss.; S. RODOTÀ, «La sovranità nel tempo della tecnopolitica. Democrazia elettronica e democrazia rappresentativa», Politica e diritto, vol. 24, n. 4 (1993), pp. 569-600; U. AllegreTtI, «Globalizzazione e sovranità nazionale», Democrazia e diritto, n. 3-4 (1995), pp. 47-66; L. FerRAjoli, La sovranità nel mondo moderno, Roma-Bari, Laterza, 1997; T. E. Frosini, Sovranità popolare e costituzionalismo, Milano, Giuffrè, 1997; A. CARRINo, Sovranità e Costituzione nella crisi dello Stato moderno. Figure e momenti della scienza del diritto pubblico europeo, Torino, Giappichelli, 1998; P. BARCELLONA, Il declino dello Stato: riflessioni di fine secolo sulla crisi del progetto moderno, Bari, Dedalo, 1998, pp. 143-155; M. R. FERRARESE, Le istituzioni della globalizzazione. Diritto e diritti nella società transazionale, Bologna, Il Mulino, 2000; N. MACCormick, Questioning Sovereignity. Law, State and Nation in the European Commonwealth, Oxford, Oxford University Press, 2002, e i più recenti N. IRTI, «Tramonto della sovranità e diffusione del potere», Diritto e società, n. 3-4 (2009), pp. 465 e ss.; A. Di Martino, Il territorio dallo stato-nazione alla globalizzazione: sfide e prospettive dello Stato costituzionale aperto, Milano, Giuffrè, 2010, e G. Silvestri, «Costituzionalismo e crisi dello Stato-nazione. Le garanzie possibili nello spazio globalizzato», Rivista trimestrale di diritto pubblico, n. 4 (2013), pp. 905-919.

36 D. Quaglioni, La sovranità, op. cit., pp. 97-116.

37 F. LANCHESTER, «L'erosione della sovranità», in F. LANCHESTER (cur.), Giuspubblicisti tra storia e politica. Personaggi e problemi nel diritto pubblico del secolo Xx, Torino, Giappichelli, 1998, pp. 155-161. 
le sviluppo tecnologico degli ultimi anni, che ha causato una ridefinizione delle tradizionali nozioni di tempo e spazio, sicché la globalizzazione finisce per tradursi, di fatto, in una relativizzazione dei confini nazionali. $\mathrm{Ne}$ consegue una realtà statale percepita come un ambito eccessivamente ristretto, e privo degli strumenti per controllare e guidare i fenomeni contemporanei, prevalentemente economici; o, di contro, troppo ampio, e pertanto inidoneo a calibrare le proprie politiche alle diverse specificità locali che la globalizzazione tende a valorizzare. La sfida che il multilevel constitutionalism pone è offrire una soluzione ai problemi che se ciascuno Stato fronteggia «individually or by simple cooperation with others has proven unable to bandle satisfactory ${ }^{38}$.

Da parti della dottrina, talora strumentali alla politica, si teorizza però che la naturale premessa del funzionamento del costituzionalismo multilivello sia la scissione di un binomio considerato fin qui indissolubile, quello tra Stato e Costituzione. L'idea di un potere diviso tra diversi livelli autonomamente legittimati e reciprocamente complementari, richiederebbe l'abbandono della concezione dell'ente statale quale cornice necessaria e preesistente all'esercizio del potere costituente e alla stessa dimensione costituzionale. Lo Stato non è più corollario alla realizzazione costituzionale di una data comunità, ma il prodotto di questa; ed è lo strumento di auto-organizzazione della società, della quale rappresenta una delle molteplici articolazioni, sebbene fondamentale. L’odierna incapacità degli Stati nazionali di concentrare l'esercizio del pubblico potere negli organi centrali potrebbe essere considerata la riprova che un assetto multilivello non solo sia già da tempo esistente nella prassi, ma che, almeno sotto la visuale dell'esercizio del potere, sia anche inevitabile.

I trattati europei rappresenterebbero la formazione di un livello sopranazionale, troverebbero fondamento nella volontà dei cittadini europei di organizzarsi secondo moduli sconosciuti al diritto pubblico tradizionale e, insieme alle costituzioni nazionali, formerebbero la Costituzione dell'Unione Europea, quale sistema unitario autolegittimantesi.

Composito contratto sociale europeo, di rousseauviana memoria, di una comunità che ha inteso organizzarsi secondo moduli nuovi, $i$ trattati sono dunque fonte di legittimazione delle istituzioni europee e dei loro atti; e sia i trattati che la normativa comunitaria risultano fortemente influenzati dalle Costituzioni nazionali degli Stati membri. D'altronde, le Costituzioni sono

${ }^{38}$ I. Pernice, «Multilevel Constitutionalism and the Treaty of Amsterdam...», op. cit., p. 715 .

Foro, Nueva época, vol. 19, núm. 2 (2016): 57-95 
di frequente state adattate agli sviluppi del processo di integrazione europea mediante procedimenti di modifica parziale. Si è in presenza di un sistema organico strutturato in diversi livelli di potere che si influenzano tra loro. I trattati, sebbene posti in essere secondo gli schemi tipici del diritto internazionale, in questa visione manifestano l'esercizio di un potere costituente da parte dei popoli europei, seppure secondo procedimenti nuovi rispetto al diritto pubblico tradizionale. La concezione degli accordi istitutivi e dei successivi emendamenti quali espressione dell'esercizio della sovranità popolare da parte dei diversi Stati membri, nei loro ambiti, confermerebbe la superiorità del diritto comunitario all'interno del sistema multilivello europeo: è proprio la comune decisione dei popoli europei di dar vita a un livello normativo superiore a fondare questa preminenza e, pertanto, tale volontà generale non potrà essere messa in discussione da un singolo Stato.

\section{LA VICENDA STORICA DEL MOVIMENTO COSTITUZIONALISTICO EUROPEO}

Secondo le regole di una relazione biunivoca, così come il costituzionalismo nasce e si afferma nell'ambito della formazione dello Stato moderno europeo ${ }^{39}$, il processo evolutivo dello Stato-nazione si snoda nel quadro della storia costituzionale. Il XIX e la prima parte del XX secolo sono stati il periodo di esaltazione dello Stato, ma la tendenza dei poteri a consociarsi, a riconoscersi reciprocamente e quindi a limitarsi è ben più risalente ${ }^{40}$. Sebbene con percorsi diversi pur nelle somiglianze, le vicende storiche degli Stati nazionali europei, che hanno portato alla maturazione del concetto di Costituzione, rispecchiano tutte una realtà che riconosce la Carta fondamentale - $\mathrm{i}$ cui necessari pilastri sono il principio democratico, la separazione dei poteri e lo Stato di diritto-, solidamente ancorata allo Stato: il fenomeno costituzionale resta legato alla dimensione statuale con un punto interrogativo aperto verso l'Europa ${ }^{41}$. Nel complesso pano-

\footnotetext{
39 «Il costituzionalismo nasce insieme allo stesso Stato moderno europeo al fine di controllare, limitare, ricondurre a regole quei poteri pubblici, che iniziarono a porsi in posizione di centralità sui territori a partire dal Quattordicesimo secolo». Si veda M. FIORAVANTI, Costituzionalismo. Percorsi della storia e tendenze attuali, Roma-Bari, Laterza, 2009, p. 5.

${ }^{40}$ M. Fioravanti, «Il modello costituzionale europeo: tradizioni e prospettive», in G. Bonacchi (cur.), Una Costituzione senza Stato: ricerca della Fondazione Lelio e Lisli Basso, Bologna, Il Mulino, 2001, p. 47.

${ }^{41} \mathrm{~S}$. Mannoni, La tradizione costituzionale in Europa. Tre itinerari nazionali tra diritto e storia: Inghilterra, Germania e Francia, Firenze, goWare, 2016, pp. 6-8.
} 
rama costituzionale europeo, campeggia l'immagine di un intero fatto di parti distinte a partire dal quale, pur senza procedere alla demolizione degli Stati nazionali, si prevede tuttavia un processo di progressiva costruzione della comune forma politica, plausibile in un futuro in cui l'Europa sia capace di esprimerla quale principio unitario costituzionalmente dato attraverso la consociazione degli stati e dei popoli, che sono le parti che compongono l'intero ${ }^{42}$.

La teoretica del costituzionalismo multilivello si propone come il melting pot di una tale unità, nel quale le costituzioni degli Stati membri e i trattati costitutivi, nonostante la loro formale distinzione, forgiano un unico e coerente sistema, nel cui ambito la competenza a esercitare il pubblico potere è condivisa tra più sistemi interdipendenti. L'Unione Europea, che è stata l'ambito nel quale il movimento costituzionalistico europeo ha avviato il percorso storico che ha registrato lo sporadico tentativo di sostituire i trattati con un testo costituzionale ${ }^{43}$, rappresenta l'espe-

${ }^{42} \mathrm{Si}$ veda in particolare il capitolo 3, La forma politica europea, parte II, in M. FIORAvanTI, Costituzionalismo. Percorsi della storia e tendenze attuali, Roma-Bari, Laterza, 2009.

${ }^{43}$ La risalente proposta per una Costituzione europea, firmata a Roma il 29 ottobre 2004, ha attraversato un difficile processo di ratifica, tanto che il 23 luglio 2007 è stata convocata una nuova conferenza intergovernativa a Lisbona per trovare un'alternativa al trattato costituzionale. Il trattato di Lisbona, firmato il 13 dicembre 2007, è entrato in vigore il $1^{\circ}$ dicembre 2009, dopo essere stato ratificato da tutti i paesi membri secondo le rispettive norme costituzionali. La maggior parte delle riforme istituzionali e politiche previste dalla Costituzione del 2004 sono riprese ma presentate in forma diversa. Il trattato costituzionale doveva abrogare i trattati istitutivi dell'UE e sostituirli con un testo unico, ovvero la Costituzione per l'Europa. Di contro, il trattato di Lisbona non sostituisce i trattati istitutivi, ma li modifica soltanto, come avevano già fatto in passato i trattati di Amsterdam e di Nizza. Tale cambiamento formale non ha conseguenze sul piano giuridico, ma è fortemente simbolico e politico. L'idea di una costituzionalizzazione dell'Europa viene abbandonata e il diritto europeo continua ad essere regolato da trattati internazionali. L'Unione è quindi ancora basata su due trattati istitutivi: il trattato sull'UE e quello che istituisce la Comunità europea (ora rinominato «trattato sul funzionamento dell'UE»). Il trattato di Lisbona riforma le istituzioni e migliora il processo decisionale e la dimensione democratica dell'UE; ne riforma le politiche interne e ne rafforza la politica esterna. Con riguardo alle questioni istituzionali, il trattato modifica le regole riguardanti la composizione della Commissione, del Parlamento europeo, del Comitato delle regioni e del Comitato economico e sociale europeo. Crea poi due nuove funzioni nell'architettura istituzionale dell'UE: il presidente del Consiglio europeo e l'Alto Rappresentante per gli affari esteri e la politica di sicurezza che rappresenta L'Unione nella scena mondiale. Abolisce la vecchia struttura a pilastri e procede a una nuova ripartizione delle competenze tra l'UE e gli Stati membri; semplifica inoltre le procedure legislative e la tipologia degli atti giuridici adottati nell'UE; conferisce una maggiore flessibilità al suo funzionamento; istituisce diverse clausole istituzionali volte ad agevolare la costruzione europea in determinati settori politici. Uno degli obiettivi del trattato di Lisbona è rafforzare la democrazia europea, soprattutto al fine di conferire legittimità alle decisioni e ravvicinare l'UE ai suoi cittadini. Sono quindi considerevolmente rafforzati i 
rimento istituzionale ove più rileva tale interdipendenza; i vari livelli di governo dell'articolato sistema di potere, siano essi sopranazionale, statale o regionale, riflettono le identità politiche dei cittadini interessati.

Dopo una lunga fase che ha visto la necessaria configurazione delle corrette relazioni costituzionali tra carte e corti dei diritti, si intravedono sia i primi risultati dell'interazione tra Costituzioni nazionali, Convenzione europea dei diritti dell'uomo e Carta europea dei diritti fondamentali dell'Unione Europea, che gli effetti generati dal sistema multilivello. Lo sviluppo della tutela sovranazionale sembra aver innescato un benefico dinamismo giurisprudenziale, che ha talora modificato orientamenti pregressi, sfociando in «una convergenza interpretativa tra le varie giurisdizioni a maggior tutela dei diritti individuali; altre volte si è invece espresso in divergenze giurisprudenziali, alcune delle quali restano tuttora irrisolte» ${ }^{44}$. I confini tra la Carta dei diritti dell'UE e le Costituzioni nazionali sono tuttora in via di definizione, e, sia pure in costanza di un atteggiamento pragmatico della giurisprudenza europea, il problema della delimitazione dell'ambito di applicazione della Carta è correlato a quello della sua diretta applicabilità ${ }^{45}$. E' utile però ricordare l'importanza che, nella pratica, va assumendo la Convenzione per i diritti umani nel sistema europeo di governance multilivello, promuovendo una sempre maggiore integrazione

poteri del Parlamento europeo. Il trattato di Lisbona attribuisce altresì un ruolo più importante ai parlamenti nazionali in seno all'UE; istituisce il diritto d'iniziativa, che permette ai cittadini di partecipare più attivamente alla costruzione dell'Europa. Uno dei cambiamenti più importanti riguarda lo spazio europeo di libertà, sicurezza e giustizia: il trattato di Lisbona rafforza le competenze dell'UE per il controllo alle frontiere, l'asilo e l'immigrazione; la cooperazione giudiziaria sia in materia civile che penale; ne rafforza la politica esterna e l'azione internazionale. L'UE acquista la personalità giuridica necessaria per negoziare ed essere parte contraente nei trattati internazionali. Infine la politica di sicurezza e di difesa comune è oggetto di una sezione in seno ai trattati istitutivi, e gli obiettivi a lungo termine sono volti ad attuare una difesa europea comune. Si veda «Il trattato di Lisbona: Introduzione», Eur-Lex, Access to European Union Law, disponibile in bttp://eur-lex.europa.eu/ legal-content/IT/TXT/?uri=URISERV por 1003Aai0033.

${ }_{44} \mathrm{M}$. CARTABIA, La tutela multilivello dei diritti fondamentali. Il cammino della giurisprudenza costituzionale italiana dopo l'entrata in vigore del Trattato di Lisbona. Incontro trilaterale tra le Corti costituzionali italiana portoghese e spagnola Santiago del Compostela 16-18ottobre 2014, Corte Costituzionale Italiana, pp. 1-21, in particolare p. 2.

${ }^{45}$ «Quali disposizioni della Carta UE e in quali circostanze godono della carattere dell'effetto diretto? Questo interrogativo [...] è di primaria rilevanza, considerato che dalla risposta che ad esso si dà dipende anche la configurazione dei rapporti, all'interno degli ordinamenti nazionali, tra giudici ordinari e Corte costituzionale. Sia il tema dell'ambito di applicazione sia quello degli effetti diretti delle disposizioni della Carta [...] sono dunque terreni di lavoro, tanto per i giudici europei, quanto per le Corti costituzionali nazionali, in vista di una più precisa definizione dei rapporti tra i diversi livelli di tutela dei diritti e tra le diverse giurisdizioni». Si veda ibid., p. 9. 
democratica ${ }^{46}$, specie nei casi di antinomie tra il diritto interno e le norme europee $^{47}$. Poiché è ormai consolidato che i diritti garantiti dalla Convenzione, che derivano dalle tradizioni costituzionali comuni agli Stati membri, siano principî generali del diritto dell'Unione, la giurisprudenza delle Corti europee sta da tempo orientando quella delle diverse Corti Costituzionali nazionali in materia di tutela dei diritti fondamentali, con effetti di sensibile innovazione del sistema, determinando alcune trasformazioni giurisprudenziali ${ }^{48}$.

Un'elaborazione dei concetti di sovranità e di Stato nazionale rispetto al costituzionalismo multilivello ha richiesto un percorso atto a superare la nozione dell'esistenza di un unico livello auto-legittimato e, in quanto tale, dominante rispetto agli altri livelli esistenti, e, nello stesso tempo, l'abbandono della concezione del pubblico potere quale autorità gerarchicamente e rigidamente organizzata. Lo stesso concetto di Costituzione è stato posto al di fuori della cornice giuridica dello Stato.

La dottrina del costituzionalismo multilivello ha elaborato negli anni un sistema nel quale convivono diversi centri di autorità formalmente distinti e autonomi gli uni dagli altri, capaci di trovare in se stessi una propria legittimazione. Da tale legittimazione discendono, tendenzialmente, l'equiordinazione dei vari livelli e l'assenza di modelli organizzativi e funzionali di tipo gerarchico.

I diversi livelli, tuttavia, si sono andati caratterizzando come fortemente interdipendenti, sia da un punto di vista strutturale che funzionale. Gli organi dell'Unione Europea sono difatti titolari di competenze e agiscono secondo regole fissate dai trattati, ma nella loro formazione, come nel funzionamento, possono dipendere in larga parte dalle disposizioni presenti nei testi costituzionali e nelle normative primarie degli stati membri. Questo assunto non riguarda soltanto i vertici dell'UE, come il parlamento o il Consiglio d'Europa, ma i legislatori, le amministrazioni, i giudici naziona-

${ }^{46}$ J. García Roca, «The Preamble. The Convention's Hermeneutic Context: A Constitutional Instrument of European Public Order», in J. García Roca e P. SAntolaya, Europe of Rights: a Compendium on the European Convention of Human Rights, Leiden, Brill, 2012, pp. 1-2 e 11-15. Riguardo alla interpretazione dei diritti costituzionali dalla prospettiva dei trattati internazionali, si veda altresì lo studio collettivo coordinato da J. GARCía ROCA e P. A. FERNÁNDEZ-SÁNCHEZ (curr.), Integración europea a través de derechos fundamentales: de un sistema binario a otro integrado, Madrid, Centro de Estudios Políticos y Constitucionales, 2009.

47 J. GARCía RoCA, «Del diálogo entre los tribunales constitucionales y las cortes supranacionales de derechos: algunas cuestiones para disputar», in R. CANOSA USERA e J. RODRíGUEZARANa MuÑoz (curr.), Jurisdicción de la libertad en Europa e Iberoamérica, Madrid, Reus, 2014, pp. 121-164.

${ }_{48}$ M. CARTABIA, La tutela multilivello dei diritti fondamentali..., op. cit., pp. 9-10. 
li chiamati a operare quali organi comunitari di implementazione e di esecuzione della normativa europea. In questo caso la compenetrazione tra $\mathrm{i}$ diversi livelli è evidente e direttamente collegata alla particolare struttura dell'Unione, che appare appunto l'ordinamento all'interno del quale l'esercizio del pubblico potere, in particolare di quello amministrativo, opera secondo criteri di effettività, con la preferenza verso le articolazioni maggiormente vicine ai cittadini.

Dal punto di vista degli Stati membri, quando l'Unione presenta competenze esclusive, o nei casi di competenza concorrente (nei quali il livello europeo appare il più idoneo a disciplinare specifiche questioni), il raggiungimento degli obiettivi nazionali finisce per dipendere dalle istituzioni e dalle procedure adottate nel proprio ambito. Gli stessi testi fondamentali dei diversi livelli sono strettamente collegati e reciprocamente influenti. In effetti, il numero e la rilevanza delle modifiche approvate nei testi costituzionali degli Stati membri in relazione al processo di integrazione europea, comporterebbe la necessità di fare riferimento a costituzioni euro-francese, euro-tedesca, euro-italiana e via di seguito ${ }^{49}$.

Peraltro, come ogni scuola di pensiero ormai di lungo corso, l'idea di una multilevel governance contiene in sé i germi del suo superamento, e questo non può che andare a detrimento del costituzionalismo multilivel$1 \mathrm{l}^{50}$, specie se, nel contesto generale, si assiste, al livello interno dei singolo stati membri, all'avvio di iter relativi a impegnativi progetti di riforma costituzionale ${ }^{51}$.

Ricordiamo che nella sua concezione classica, prescrittiva, la Costituzione, come si è detto, è stata sempre considerata, fin dalle prime elaborazioni teoriche, quale nozione strettamente connessa alla concettualizzazione dello Stato nazionale ${ }^{52}$ e, nella prassi, concepita per durare a lungo.

49 A. MAnZELla, «Principio democratico e integrazione europea», Quaderni Costituzionali, n. 3 (2006), p. 569.

${ }_{50}$ A. CANTARO, «Il superamento della multilevel theory nei rapporti tra l'Europa, gli Stati membri e i livelli di governo sub-statale», in S. MANGIAMELI (cur.), Governance europea tra Lisbona e Fiscal compact, Milano, Giuffrè, 2016, pp. 119-131.

${ }_{51} \mathrm{Si}$ fa riferimento, in particolare, al caso italiano. La riforma approvata dal Parlamento il 12 aprile 2016, ora sottoposta a referendum popolare, unitamente ai contenuti essenziali della nuova legge elettorale per la Camera dei deputati e alla disciplina normativa che regola il referendum sulle revisioni costituzionali, qualora entri in vigore sembra destinata a innovare in profondità l'impianto istituzionale della Repubblica italiana. P. Bilancia e F. G. PizzeTTI, Testi e progetti del sistema costituzionale italiano ed europeo. Edizione speciale per il Referendum costituzionale, Torino, Giappichelli, 2016.

52 M. LuCIANI, «L'antisovrano e la crisi delle Costituzioni», Rivista di diritto costituzionale, n. 1 (1996), pp. 124-188, in particolare p. 126. 
$\mathrm{Al}$ di là della nozione assiologica ${ }^{53}$, s'è andata consolidando una recezione dell'idea di Costituzione parzialmente diversa, di matrice giuspositivistica e formalistica. In essa l'aspetto contenutistico e politico-ideologico perde ogni rilevanza a favore di elementi formali, quali la forma scritta e il suo essere legge superiore, che la caratterizzano come testo costituzionale. Irrilevante, in questa impostazione, è la cornice di valori e principî in cui essa si inserisce e di cui è portatrice, come il fatto che abbia o meno derivazione popolare: la validità di una Costituzione si fonda solo sulla sua effettività. Secondo tale accezione la nozione di Costituzione smarrisce quindi il suo significato più profondo, quello di legittimazione e di limitazione del potere politico. Essa però continua a presentare la caratteristica di essere strettamente legata alla nozione di Stato, in quanto il suo essere legge fondamentale implica l'esistenza di una organizzazione capace di assicurarne l'applicazione e l'osservanza e quindi, in una parola, sovrana. Nella dottrina contemporanea, la scelta dell'una impostazione rispetto all'altra è, in definitiva, una scelta di valore ${ }^{54}$.

Peraltro se la teoria del costituzionalismo multilivello ha trovato negli studi sulla crisi dello Stato nazionale la sua cornice teorica, deve essere messo in evidenza come le scuole di pensiero che prevedono, o auspicano, la fine della sovranità nazionale, e con essa delle entità statali (in forza della globalizzazione economica, di concezioni estreme della democrazia pluralista, nonché universalistiche dei diritti fondamentali e della dignità umana), pronosticano la fine dello Stato-nazione, ma, curiosamente, non anche della Costituzione. Magna pars della dottrina, difatti, non critica la sopravvivenza delle costituzioni nazionali ${ }^{55}$. Un'altra parte richiede, sulla base dell'universalismo dei diritti, una Costituzione mondiale che abbia nell'umanità intera il proprio referente ${ }^{56}$. Altra ancora discute di un governo della società mondiale trasferito a strutture a rete, con terminali rappresentati da livelli di diverso peso tra loro interdipendenti e capaci di rispon-

53 Ricordiamo che i valori supremi, quali obiettivizzati nella coscienza dei popoli che si danno le costituzioni positive, fungono da architravi degli ordinamenti giuridici, e sono «il portato della cultura epocale di un popolo o - più esattamente ai nostri giorni- dei popoli che hanno scelto di passare dal fondamento di autorità a quello di valore». L'organizzazione democratica della quale il popolo è parte consegue sempre alla premessa assiologica originaria. Si veda G. SILVESTRI, «La dignità umana come criterio del bilanciamento dei valori costituzionali», in A. D'Atena (cur.), Studi in onore di Pierfrancesco Grossi, Milano, Giuffrè, 2012, pp. 1179-1180.

54 A. Anzon, «La Costituzione europea come problema», Rivista Italiana di diritto pubblico comparato, n. 3-4 (2000), p. 636.

${ }_{55}$ G. Zagrebelsky, Il diritto mite, Torino, Einaudi, 1992, pp. 7 e ss.

${ }^{56}$ L. FerRAJOli, La sovranità nel mondo moderno, op. cit., pp. 50 e ss. 
dere in modo flessibile alle nuove esigenze e ai nuovi interessi emergenti, abdicando tuttavia alla nozione di Costituzione in senso prescrittivo ${ }^{57}$.

Risulta evidente come, nella problematica della Costituzione europea, né il modello di una Costituzione mondiale né quello ipotizzato della concezione postmoderna, fondato su rapporti e istituzioni reticolari, offrano soluzioni valide. Le due teorizzazioni non conseguono la definizione di una nozione giuridica di Costituzione, in quanto deboli sul piano dell'effettività, e vuote dell'analisi del problema degli strumenti istituzionali finalizzati a disciplinare l'uso del potere e la realizzazione dei valori incorporati nel testo costituzionale ${ }^{58}$. La discussa prospettiva di un possibile governo mondiale, pone infine, anche a livello sovranazionale, il problema del limite $\mathrm{a}$ un potere arbitrario e la necessità di delineare un nuovo modo di concepire il «pubblico» sia nei sistemi di civil law che di common law.

A questo punto, s'impone la riflessione sull'emersione dei valori giuridicamente tutelati e sulla protezione dei diritti fondamentali, e su come l'esperienza costituzionale europea li enunci e difenda, rielaborandoli per qualificarli come principî d'una tradizione costituzionale comune ${ }^{59}$. Come contrastare il diffuso scetticismo che nega vitalità alla condizione antropologica e culturale conseguente al proclamato tramonto della modernità nelle società del capitalismo maturo, che riguarda il presente e sarà il problema del futuro? In che modo il contemporaneo postmodernismo potrebbe ritrovare l'energia positiva che deve animare qualsiasi progetto di costruzione istituzionale, sia pure solo teorico? E, nell'estremo tentativo di superamento dell'impasse, come recuperare il pensiero critico che promuove i cambiamenti?

Alla rivoluzione culturale degli anni Sessanta, si sono avvicendati l'austerity dei Settanta, l'edonismo yuppie degli Ottanta, il collasso sovietico e la fine della «prima repubblica» italiana dei Novanta, il tramonto malinconico del secolo breve e il crollo delle ideologie del Duemila. I radicali e vorticosi mutamenti del mondo occidentale ripropongono l'interrogativo comune a tutte le cesure storiche: la nostra cultura è in crisi o in decadenza? Non è solo una questione terminologica; da una crisi si può uscire, mentre la decadenza potrebbe essere senza ritorno. In un contesto poco vivace, nel quale il superficiale, l'attuale e l'episodico obliterano i significati

${ }^{57}$ L. PINelli, «Cittadini, responsabilità politica, mercati globali», Rivista di Diritto Costituzionale (1997), pp. 64 e ss.

${ }_{58}$ M. LuCIANI, «L'antisovrano e la crisi delle Costituzioni», op. cit., pp. 174 e ss.

59 E. Triggiani, «La complessa vicenda dei diritti sociali fondamentali nell'Unione europea», Studi sull'integrazione europea, n. 9 (2014), pp. 9-33, in particolare p. 29. 
profondi della vita e minano le radici stesse della coesistenza civile, la decadenza è testimoniata dal trionfo dell'effimero, che nega le ragioni dell'etica e della politica e ostruisce i percorsi del diritto e della legalità. E, soprattutto, svilisce la funzione della cultura, unica via di superamento della condizione presente, nella quale si considerano le facoltà critiche sopravvalutate e non necessarie; anzi, quel che caratterizza la nostra epoca, è proprio il loro spreco. La perdita di criticità, e tout court di intelligenza, nell'affrontare ogni questione, è un danno per l'intera società, condannata all'appiattimento e svuotata del bene che è la diversità. Si procede, accelerando, nell'inesorabile discesa verso il basso capitalismo, o, per usare un icastico neologismo, verso la catamodernità ${ }^{60}$.

Nella crisi del capitalismo globalizzato, la modernità, tuttavia, con il suo immenso portato razionale, non sa morire: o, almeno, lo si spera. La resuscitata polemica modernità-postmoderno è peculiare dell'attuale situazione culturale; ne manifesta la precarietà con il nostalgico atteggiamento verso il passato, l'incertezza sui compiti presenti e il buio riguardo alle aspettative per il futuro. «Il postmoderno si ritrae, filosoficamente e ideologicamente, non perché abbia mancato i suoi obiettivi ma, proprio al contrario, perché li ha centrati fin troppo bene. Ciò che hanno sognato i postmoderni l'hanno realizzato i populisti, e nel passaggio dal sogno alla realtà si è capito davvero di che cosa si trattava» ${ }^{61}$. Nel dilagare del populismo, che non è solo un rischio per la politica, ma può divenire una malattia della democrazia ${ }^{62}$, l'uomo dell'età catamoderna è un vinto di malavogliana memoria: vive la sconfitta della modernità, delle sue utopie e delle sue

${ }^{60}$ Come afferma Francesco Muzzioli nel suo geniale Manifesto della catamodernità, questa è così definita «non solo perché il cata- è il prefisso della catastrofe, nelle cui vicinanze ci dibattiamo. Anche perché il prefisso cata- indica una caduta verso il basso e quindi si attaglia alla fase di "basso Capitalismo" (come si dice "basso Medio Evo") in cui il nostro stesso continente sembra condannato a un innarrestabile declino». Si veda F. Muzzioli, «Manifesto della catamodernità», in Verbigerazioni catamoderne. Con un sussidiarietto di lettura di Marcello Carlino, Pescara, Tracce, 2012, pp. 123-127. Se la realtà è infinitamente manipolabile, la verità è una nozione inutile: in questo assioma si concretizza il pensiero postmoderno, che ha pervaso ogni ambito della quotidianità, dalla politica, all'arte, alla letteratura, alla dipendenza dal linguaggio delle fiction e dei reality, così finto da sembrare vero: ecco perché siamo precipitati a capofitto nella catamodernità. E se il postmoderno è il processo che ha liquidato la modernità e il suo criticismo radicale, il catamoderno, in contrapposizione ad esso, intende estremizzare la modernità, che è affondata, consapevole della contradditorietà dell'essere e della multiformità del reale, ma forse è ancora percorsa da una seppur minima scintilla vitale.

${ }^{61} \mathrm{Si}$ veda in particolare il primo paragrafo, «Dal postmoderno al populismo», del capitolo 1, Realytismo. L'attacco postmoderno alla realtà, del saggio di M. FERRARIS, Manifesto del nuovo realismo, Roma-Bari, Laterza, 2014.

${ }^{62}$ L. Violante, «Appunti per un'analisi del populismo giuridico», Democrazia e diritto, n. 3 (2010), pp. 107-125. 
conquiste. Il concetto di catamoderno, però, non contiene soltanto un'amara diagnosi dello stato delle cose, ma implica un imperativo: la necessità di far rivivere la modernità e i suoi profondi significati di progresso, per un risveglio dall'attuale sonno della ragione che omologa il pensiero, esclude la critica, e respinge la possibile rivalutazione dei fini comuni. Per raggiungere tali fini —quelli della convivenza civile, della solidarietà, del perseguimento della legalità-, occorrerebbe che fosse comune anche un percorso. La meta del cambiamento non può essere un ritorno al passato vuoto di efficacia concreta. Per cambiare il mondo, al contrario, occorre sempre un atto politico (nel primitivo senso di «relativo alla polis») reale e collettivo, col pre-requisito della consapevolezza critico-dialettica del presente. Altrimenti, ogni buona intenzione diventa utopia. E non vi è più una moderna stella polare che guidi l'atteggiamento razionale dell'uomo; siamo circondati da cattivi maestri ma orfani di Maestri; postmoderne variabili di riferimento comportamentale, culturale o sociale, rendono l'azione umana incerta, problematica. L'uomo ha sempre la possibilità di abbandonare la lotta finalizzata a migliorare il proprio stato e fuggire, ma la fuga non potrà avvenire in una direzione qualsiasi. Meno che mai in avanti. E tornando indietro nel tentativo di recuperare vecchi modelli per fondare una nuova polis, si rischia di perdere la connessione col reale, di non sapere più che cosa sia il mondo. Un mondo in cui impera l'assurdo, che ha sconfitto i sogni e azzerato la progettualità, nel quale la condizione umana spesso si identifica nel contrasto fra persecutori e perseguitati. Ecco quindi la necessità di spostare la conflittualità materiale alla conflittualità sul terreno delle idee con la determinazione di risolverli, i conflitti, mediante una rinascita dell'intelligenza e della critica, per rilanciare una nuova modernità ${ }^{63}$. Nell'orizzonte del mito che ha governato la coscienza per millenni, si delinea il fondamento teorico del diritto dell'uomo a un destino felice, che gli compete in forza della dignità della sua natura, e che preme perché l'esistenza trovi nuove forme e nuove modalità di espressione e che in tali forme incontri il futuro. Un nuovo Illuminismo implica fede nell'uomo e nel progresso, e, per il rinnovo della politica, il recupero di un progetto

${ }^{63}$ La sfida catamoderna è l'ultimo, maestoso manifesto di un movimento forte d'una spontaneità urgente che decodifica la catastrofe e il conseguente bisogno di arginare l'avanzata del nulla, che nobilita l'ansia di riempire i vuoti d'una esistenza trascorsa sui social, tra post e selfie, con il libro, il viaggio, l'arte, e ogni processo creativo possibile, sia pure in fieri. Si consenta il rinvio a R. AlibRand, «Elogio del catamoderno. Esegesi di una condizione», Le idee, Live Sicilia (2016), disponibile in http://livesicilia.it/2016/05/22/ elogio-del-catamoderno_750963. 
comune. E l'idea d'Europa, fin qui, è stata il grande progetto comune che ha aggregato popoli diversi.

\section{PRIMA CHE SIA TROPPO TARDI. IL RECUPERO DEL SIGNIFICATO STORICO DELLA VOCE «COSTITUZIONE»}

Ritornando all'analisi del costituzionalismo europeo, si rileva tuttavia che, al di là dei quadri teorici ideali, la nozione cui si fa riferimento, almeno implicitamente, è quella di Costituzione prescrittiva. L'impostazione dottrinale classica prevede un testo scritto, normativo, tendenzialmente stabile, assiologicamente orientato, fondativo e limitativo del potere. Resta da stabilire se l'Europa possa dotarsi d'una Carta che contempli diritti fondamentali e interessi comuni, costellazione di valori che fa capo alla dignità umana e afferma e preserva la propria sovranità «solo per effetto di un'azione incrociata tra sovranità nazionale e potestà sovraordinata dell'ordinamento internazionale e degli ordinamenti sovranazionali» ${ }^{64}$.

Dalla discrasia tra una autorità a livello europeo in crescita e una legittimazione democratica ancora circoscritta al solo ambito statale deriva un deficit democratico irrisolto. A questo consegue l'impossibilità di pensare una Costituzione europea, data l'assenza di un popolo europeo (perché, ricordiamolo, non esiste altra sovranità se non quella che promana dal popolo e una Costituzione promana dal potere costituente del popolo), cui imputare un atto costituente: $\mathrm{i}$ trattati rappresenterebbero testo costituzionale dato da terzi e non disponibile da parte dell'Unione. Una parlamentarizzazione piena dell'Unione sul modello di quanto avviene negli Stati costituzionali non è possibile né auspicabile nel breve periodo, in quanto essa finirebbe per incrementare il problema del deficit democratico, allentando il vincolo dell'Unione verso gli Stati membri e concentrando, piuttosto, i poteri in un organo, il parlamento europeo, che rimarrebbe comunque non rappresentativo del popolo europeo, non esistente come tale, in atto, come entità omogenea ${ }^{65}$.

Se il demos costituisce la sua legge, la legge costituisce il suo demos. Nel caso europeo si tratta di un'opera titanica ${ }^{66}$ : l'identità europea non può

${ }^{64}$ G. Silvestri, «La parabola della sovranità...», op. cit., p. 83, e ID., G. SilvestrI, «Verso uno ius commune europeo dei diritti fondamentali», op. cit., pp. 7-11.

${ }^{65}$ P. GRIMM, «Does Europe Need a Constitution?», op. cit., pp. 359-361.

${ }^{66}$ E. RESTA, «Demos, Ethnos. Sull'identità europea», in G. BonAcCHI (cur.), Una Costituzione senza Stato: ricerca della Fondazione Lelio e Lisli Basso, Bologna, Il Mulino, 2001, p. 187. 
essere la mera trasposizione politica di una realtà storicamente consolidata. A sua volta, sarà determinata da un processo che filtri le innumerevoli possibilità esistenti nel quadro europeo ${ }^{67}$. Lo Stato nazionale, così come costituitosi nel XIX secolo, era il risultato di un lungo processo; il contenitore atto a definire uno spazio e un terreno comuni, costituendo la sovranità democratica per l'azione di quel soggetto collettivo in senso proprio che è il popolo ${ }^{68}$, e che ha manifestato un'attenzione a tale spazio, e alla definizione di sé, funzionale alla necessità di mostrare la nuova formazione politica indipendente quale entità compiuta e distinta dalle altre, con la precisazione dei limiti visibili e degli ambiti riconoscibili, che a esso sottendono. Seppure si officiano da più parti i funerali dello Stato-nazione e, con riguardo al caso italiano, si rileva la perdita di sovranità subita dall'Italia nello scenario dell'Unione Europea, è opportuno sottolineare come, fino a tempi recenti, tali questioni fossero prevalentemente giuridiche; la crisi imperante le ha invece trasmutate nella sfera politica. La limitazione della sovranità dello Stato, in passato, era di certo ritenuta possibile, tanto da essere costituzionalmente prevista, ma solo in funzione di superiori esigenze di pace e sicurezza, poiché i costituenti ritenevano che i benefici in tal modo conseguiti avrebbero ampiamente compensato le eventuali autolimitazioni adottate ${ }^{69}$. E in un'era critica per tale sovranità, è sempre attuale il problema del fondamento delle garanzie dell'individuo nello spazio globale; nell'esperienza costituzionale europea la tutela dei diritti fondamentali deve tenere un ruolo di primo piano ${ }^{70}$.

Non sembra pertanto che l'Europa possa seguire il percorso secolare degli Stati nazionali, specie se costruita su un principio di libertà e sulla garanzia dei diritti. Questa caratterizzazione sfuma i suoi confini e fa sì che lo spazio europeo sia in continua ridefinizione. Se il popolo europeo è una società di estranei giuridicamente fondata, le problematiche poste non sono legate allo Stato, né l'impulso allo sviluppo delle garanzie costituzionali promana da esso, ma, al contrario, dalla necessità di limitare il potere politico istituzionalizzato nella forma giuridica dello Stato, quale storicamente configurata nelle diverse esperienze nazionali ${ }^{71}$.

${ }^{67}$ P. CostA, «La cittadinanza fra Stati nazionali e ordine giuridico europeo: una comparazione diacronica», in G. BONACCHI (cur.), Una Costituzione senza Stato: ricerca della Fondazione Lelio e Lisli Basso, Bologna, Il Mulino, 2001, pp. 307 e ss.

${ }^{68}$ Cfr. G. Preterossi, Ciò che resta della democrazia, Roma-Bari, Laterza, 2016, pp. 96-102.

${ }^{69}$ G. SCARPARI, «Sovranità limitata», Il Ponte, vol. 72, n. 5 (2016), pp. 5-13, in particolare p. 5.

${ }^{70}$ G. Silvestri, «Costituzionalismo e crisi dello Stato-nazione...», op. cit., pp. 914 e ss.

${ }^{71}$ Poiché l'idea di Costituzione in senso prescrittivo nella sua concettualizzazione cor- 
Come ricordato ${ }^{72}$, i trattati europei non hanno le caratteristiche di una Costituzione democratica, né è possibile conseguire una Carta costituzionale mediante le procedure del diritto internazionale, cha la depriverebbero, se si ergessero a potere costituente, della necessaria legittimazione popolare; è peraltro evidente che i testi dei trattati non esprimano alcuna volontà esplicita di proporsi come Costituzione. Redatti nella forma tipica dei trattati internazionali, quali patti tra Stati rimandano a conferenze intergovernative per la loro modifica e richiedono la ratifica unanime dei membri contraenti secondo le rispettive norme costituzionali per la loro entrata in vigore ${ }^{73}$. Inoltre, secondo i canoni della dottrina classica, la Costituzione deve essere la risultanza di un processo democraticamente fondato, di un potere costituente popolare, per cui i trattati, non derivando dalla volontà espressa dal popolo europeo ma dagli Stati, non possano essere considerati una Costituzione ${ }^{74}$.

Il fascino della visione della Carta di Nizza quale nucleo di un processo costituente deriva dalla considerazione che la Carta è parsa creare le premesse per una legittimazione democratica dell'Europa. Se oggi non è possibile parlare di un popolo europeo, questo potrebbe diventare fattibile in un futuro, certo non vicinissimo, proprio grazie ai diritti. La loro tutela, la coscienza di essi, creano le condizioni per l'apertura di uno spazio pubblico europeo e per la creazione di un demos europeo ${ }^{75}$. Sarà poi questo a dare piena legittimazione all'Unione secondo i canoni della dot-

risponde alle carte rivoluzionarie del secolo xviII e alle costituzioni democratiche del secolo XX, ma è, invece, lontana dai documenti costituzionali «concessi» del secolo XIX, quali, ad esempio, lo Statuto Albertino (che pur prevedendo un catalogo dei diritti, una camera elettiva e il principio della separazione dei poteri, non è riconducibile all'esercizio di un potere costituente di derivazione popolare, ma è il prodotto di una concessione regia), si è parlato, in linea analogica, di una Costituzione europea come realtà che potesse nascere in modo «ottriato». Si veda A. RUGGERI, «Sovranità dello Stato e sovranità sopranazionale, attraverso i diritti umani e le prospettive di un diritto europeo "intercostituzionale" », in Itinerari di una ricerca sul sistema delle fonti. V Studi dell'anno 2001, Torino, Giappichelli, 2002, p. 222.

${ }^{72} \mathrm{Si}$ fa riferimento alle note 19, 22, 25 e 41 al presente testo.

73 A. Anzon, «La Costituzione europea come problema», op. cit., p. 651.

${ }^{74}$ M. LuCIANI, «Legalità e legittimità nel processo di integrazione europea», in G. Bonacchi (cur.), Una Costituzione senza Stato: ricerca della Fondazione Lelio e Lisli Basso, Bologna, Il Mulino, 2001, p. 71 ss; si veda anche V. LipPoLIS, «La cittadinanza europea», Quaderni Costituzionali, n. 1 (1993), p. 131.

75 Riguardo all'obiettivo della realizzazione di una comunità politica fondata sulla realizzazione dei diritti fondamentali e sulla loro effettività nell'ambito di una protezione dei medesimi anch'essa «multilivello», si veda l'attualissimo S. GAMBINO, «Vantaggi e limiti della protezione multilevel dei diritti e delle libertà fondamentali, fra diritto dell'Unione, convenzioni internazionali e costituzioni nazionali», in A. RugGERI (cur.), Scritti in onore di Gaetano Silvestri, t. II, Torino, Giappichelli, 2016, pp. 1054-1067. 
trina costituzionale classica, benché in forme e in forza di modalità probabilmente nuove. In sostanza, nelle more di tale legittimazione, i diritti si configurano come lo strumento più adeguato a costituire un ideale equilibrio, in un sistema di pesi e contrappesi. Nella visione della società aperta degli interpreti, in cui chi vive la norma indirettamente contribuisce alla sua interpretazione e quindi alla sua attuazione, essi assurgono a chiave di volta della legittimazione democratica europea al di fuori dei processi elettivi ${ }^{76}$.

\section{IL COSTITUZIONALISMO MULTILIVELLO NELL'IPOTESI D'UNA COSTITUZIONE EUROPEA}

Nell'ottica del costituzionalismo multilivello i trattati, fin dal Trattato di Roma, rappresentano la Costituzione in senso materiale dell'Europa non meno delle costituzioni nazionali; come queste ultime, essi originano e traggono legittimazione dalla volontà dei cittadini. Ogni evoluzione dell'Unione Europea, anche se espressa in forme sconosciute al costituzionalismo classico (come i trattati, che sono negoziati e conclusi nella forma di un trattato internazionale), rappresenta in primo luogo una scelta dei suoi cittadini, i soli legittimati a decidere sulla divisione delle competenze tra la Comunità e gli Stati membri, sulle revisioni nella struttura dell'Unione medesima, o su qualsiasi altra questione che inerisca ai valori e ai diritti comuni ai popoli degli Stati membri.

In questa luce, i trattati istitutivi, quali modificati dal Trattato di Lisbona, possono essere interpretati come espressioni del contratto sociale europeo, come strumento attraverso il quale i popoli dell'Europa unita definiscono il proprio status di cittadini dell'Unione europea.

L'esistenza di un unico sistema integrato e interdipendente comporterebbe l'impossibilità di parlare di costituzioni nazionali quali elementi autonomi e indipendenti rispetto al fenomeno dell'integrazione europea.

Parte della dottrina ha così proposto l'esplicito riferimento alla matrice europea di testi che ormai, dati i cambiamenti intercorsi in ragione del processo di unificazione del Vecchio Continente, non posso più essere considerati meramente nazionali ${ }^{77}$.

76 P. HäBERLE, «Potere Costituente (teoria generale)», in Enciclopedia Giuridica, vol. XXIII (2000), pp. 8 e ss.

77 A. ManZella, «Principio democratico e integrazione europea», Quaderni Costituzionali, n. 3 (2006), pp. 569 e ss. 
Si tratta di una posizione rivoluzionaria rispetto ai tradizionali modi di intendere il costituzionalismo. Parlare di processo costituzionale in riferimento all'esperienza europea è stato considerato per lungo tempo una provocazione. Eppure nella visione post-nazionale esso include tutti gli strumenti di organizzazione e limitazione dell'autorità pubblica, in particolar con riferimento ai poteri legislativo, giudiziario e legislativo. In questo senso la Costituzione esprime quindi il consenso di individui desiderosi di dotarsi di determinate istituzioni, poteri e procedure, nonché di diritti e doveri per i quali definiscono se stessi «cittadini» di un ente che può essere un Stato, ma anche una organizzazione sopranazionale.

Le differenze rispetto alla concezione classica del costituzionalismo sono evidenti. Esse si concretizzano in particolare nel carattere non esclusivo di intendere la Costituzione e nella rottura del legame necessario che la lega allo Stato nazionale.

La prima differenza rende possibile la concettualizzazione del costituzionalismo multilivello quale sistema di condivisione del potere pubblico tra diversi livelli di autorità, nessuno dei quali esaurisce e comprende interamente tale potere; la seconda evidenzia come la preesistenza di una comunità statale non rappresenta l'elemento fondante e necessario per avere una prospettiva costituzionale. In tal senso non esiste più Stato di quanto la Costituzione ne crei.

In definitiva, sebbene le prospettive possano sembrare nel breve periodo infelici, il dibattito sul futuro dell'Europa su un piano costituzionale pare destinato a perdurare ostinatamente, in quanto permarranno le necessità materiali e simboliche che hanno contribuito ad accendere il dibattito nel decennio precedente ${ }^{78}$.

Molti cambiamenti sono intervenuti, e ancora sono in corso, nei sistemi di rappresentanza all'interno degli ordinamenti di democrazia avanzata, e mostrano una tendenza sempre più accentuata ad articolare i pubblici poteri su una pluralità di livelli di governo. Si assiste a processi di ridislocazione del potere riconducibili alla crisi dello Stato nazionale ${ }^{79}$,

\footnotetext{
${ }^{78}$ N. WALKer, «Una Costituzione con la "C" maiuscola o con la "c" minuscola?», Quaderni Costituzionali, n. 4 (2005), pp. 881-883.

${ }^{79}$ Una celebre prolusione di Santi Romano, tenuta presso l'Università di Pisa già nel lontano 1909, anticipava già i temi relativi alla decadenza dello Stato nazione, entità dotata di determinate caratteristiche, tra le quali la tendenziale ostilità a riconoscere un potere superiore, la rivendicazione per sé il monopolio della forza legittima e l'originarietà del proprio ordinamento quale condizione di efficacia e vigenza del diritto, che si ritiene comunemente sorta a partire, approssimativamente, dal Xv secolo, formalmente affermatasi nella prima metà del secolo XVII e che ha raggiunto il proprio apice nel corso del XIX, viene oggi
} 
su una pluralità di livelli come se l'ente statale progressivamente percepito quale ambito inadeguato a svolgere i compiti storicamente realizzati fosse soggetto a un processo di erosione delle sue tradizionali prerogative, nella duplice direzione dell'internazionalizzazione/sopranazionalizzazione e della localizzazione.

La nozione di costituzionalismo multilivello a cui è strettamente legata la nozione di governo multilivello, elaborata nell'ambito del dibattito sulla Costituzione europea, mette in discussione la nozione tradizionale di Costituzione della giuspubblicistica di civil law, ricondotta a due significati fondamentali: limitazione del potere e legittimazione dello stesso. L'idea di legittimazione, in relazione al costituzionalismo multilivello, induce a riflettere sulla rappresentanza politica, in quanto la possibilità di pensare una Costituzione europea, sia che essa già esista in concreto nei fatti, sia che si affermi, comunque, la necessità di un testo documentale, implica un certo modo di pensare il pubblico potere e il suo referente personale, il popolo.

Edificare una Costituzione europea significa recidere il vincolo necessario tra Stato nazionale e Costituzione cui si è fatto cenno; negare che il primo sia consustanziale rispetto alla seconda, come affermato dalla scuola statalista. Qualora se ne accetti l'idea, si deve accogliere la premessa che la Costituzione non può essere concepita come prodotto esclusivo dello Stato, in quanto entità sociologica e storica preesistente. Se questo non è, allora non può che essere il contrario: sarà lo Stato ad essere il prodotto del potere costituente. Ne consegue che il pubblico potere non solo è il prodotto della Costituzione, ma che esiste tanto pubblico potere quanto ne produce la Costituzione, la quale è in grado di distribuirlo e dislocarlo.

La visione di una Costituzione per l'Europa, infine, implica necessariamente che si concepisca il «popolo» in un certo modo: è evidente che l'Europa non abbia una unità etnica, né linguistica, culturale o religiosa. Ecco dunque che nella teoria del costituzionalismo multilivello vi è il pensiero forte del rifiuto del concetto di popolo quale entità ideale, precondi-

comunemente percepito quale soggetto sempre più refrattario ad essere inserito e interpretato secondo i paradigmi propri della costruzione idealtipica, ma storicamente orientata, della dottrina giuspubblicistica europea della metà dell' 800 . Non vi è dubbio difatti che l'esaltazione e l'assolutizzazione dello Stato nazionale attraverso la rigida identificazione tra diritto e legge parlamentare (cioè statale) e la considerazione della stessa normatività quale prodotto esclusivo della forma giuridica statale paiono oggi sempre più lontani dalla realtà di ordinamenti giuridici in profonda trasformazione. Si veda S. ROMANO, «Lo Stato moderno e la sua crisi, prolusione tenuta all'Università di Pisa nel 1909», in Lo Stato moderno e la sua crisi. Saggi di diritto costituzionale, Milano, Giuffrè, 1969, pp. 5 e ss. 
zione del pubblico potere: il popolo è inteso, piuttosto, nella sua concretezza e molteplicità, per cui un individuo è facente parte di più comunità contemporaneamente, in modo non esclusivo. In tale concezione l'identità quindi non è predeterminata e predeterminante, ma si costruisce attorno a una comune cittadinanza politica. In sostanza, se l'identità nazionale si è costruita attraverso un processo di astrazione rispetto alle precedenti identità localistiche, si assume che questo possa accadere anche fuori della cornice dello Stato nazionale.

\section{UNIONE POLITICA O COSTRUZIONE GIURIDICA? LE POSSIBILI CONCLUSIONI}

La teoria del costituzionalismo multilivello assume un connotato valoriale se si riconnette all'idea e alla struttura della rappresentanza, e si relaziona ai diversi livelli di potere enucleabili all'interno di ciascuno di essi. Tuttavia, nei sistemi di governo multilivello, rileva l'esigenza generalmente percepita di garantire che le decisioni, in particolare quelle concernenti la distribuzione delle competenze, direttamente riferibili al principio di sovranità, non siano assunte esclusivamente dal livello superiore e che, in via subordinata, esse tengano conto della disomogeneità dei panorami di riferimento e quindi delle effettive capacità del singolo ente. E' evidente la necessità di creare strumenti di raccordo tra i diversi livelli e di elaborare moduli procedimentali e organizzativi in grado di dar vita a processi decisionali partecipati.

Questa esigenza ha motivato, nel sistema italiano, le istanze di modifica del bicameralismo paritario attraverso una riforma del Senato, relativa non soltanto alla consistenza numerica e alla provenienza dei membri, ma alle prerogative dell'antica Camera Alta, che non avrà più il potere di dare o togliere la fiducia al governo, ma solo la possibilità di esprimere proposte di modifica delle leggi, la cui approvazione sarà quasi sempre prerogativa della sola Camera, con il risultato pratico d'un iter molto più rapido, e quello tacito, d'un governo ancora più forte, con una corsia preferenziale per i suoi provvedimenti, e un'espansione ulteriore a scapito del potere legislativo. Fino a oggi, in Italia si era lasciata al Senato la storica funzione di «contrappeso», al fine di consentire un corretto equilibrio costituzionale. Il ruolo di Camera di garanzia appariva essenziale a fronte del rafforzamento del rapporto tra il governo e la maggioranza parlamentare, se incentrato su una sola Camera. In tale ottica, si vedeva la funzione di garanzia 
del Senato perfettamente compatibile con quella della rappresentanza territoriale, dalla quale risulta consolidata e rafforzata.

Più da storici che da giuristi, occorrerebbe ricordare che la qualità dell'azione di governo non può essere giudicata sul piano della sua durata e della velocità dei processi decisionali, ma investe il merito delle scelte operate. E' caduta la polvere sull'idea di democrazia che la Resistenza aveva sedimentato in Italia, basata sulla più ampia partecipazione popolare, sul regime parlamentare, che proprio attraverso le sue contraddizioni, le sue «instabilità» garantiva che l'esecutivo non si affrancasse troppo dalla base elettorale?

Il sistema parlamentare e proporzionale della «prima repubblica» ha prodotto un riformismo ben più forte e incisivo di quanto non sia stato posto in essere dal sistema politico della seconda: scolarizzazione di massa, riforma del Codice di procedura penale, Statuto dei diritti dei lavoratori, divorzio, istituzione della giustizia amministrativa, nuovo diritto di famiglia, riforma sanitaria, riforma agraria, e potremmo continuare a lungo. Tutte riforme che surclassano per qualità, rendimento e durata le riformine e le controriforme del parlamento della «seconda repubblica», senza alcun possibile confronto. Ma oggi non è più sufficiente sezionare i totem nostrani per costruire ipotesi di futuro.

La dimensione europea è entrata definitivamente nell'orizzonte di azione e cognizione dei custodi delle costituzioni nazionali. Per essere protagonisti attivi di una costruzione comune, occorre perseguire l'equilibrio tra un processo di armonizzazione di base, che prevede un livello di integrazione europea che consente agli Stati il mantenimento del proprio sistema normativo in linea con i propri obiettivi sociali, e uno in fieri, nel quale il diritto europeo — anch'esso analizzabile come un sistema multilivello ${ }^{80}$-, sembra sostituirsi, in alcuni settori, a quello statale. Il cambiamento, al di là delle evidenti ricadute sul diritto privato e dell'economia, riveste una dimensione pubblica, costituzionale. Il modello a strati (Unione Europea-governi nazionali-regioni-comuni) della governance europea, nell'ambito del quale l'esercizio del potere si colloca nei punti di intersezione tra le varie istituzioni e i singoli livelli di potere, risponde a criteri di legittimazione democratica e di controllo difformi da quelli nazionali, per cui lo spostamento del livello normativo implica conseguenze anche per il sistema nazionale e gli equilibri costituzionali.

${ }^{80}$ V. S. PiatToni, The Theory of Multi-Level Governance. Conceptual, Empirical, and Normative Challenges, Oxford, Oxford University Press, 2010, pp. 17 e ss., in particolare p. 250, e C. Panara, The Sub-national Dimension of the EU..., op. cit., pp. 1-4, 44-46 e 155-158. 
In buona sostanza, l'espansione del diritto europeo necessita la creazione di meccanismi adeguati alla sua recezione ${ }^{81}$. Il processo costituzionale europeo dovrebbe connotarsi come sperimentazione corale di un modello di governance che si configuri come partecipativa, e che, coinvolgendo ampiamente la società civile, sia direttamente deliberativa, ai fini di una crescente legittimità delle decisioni prese ${ }^{82}$. Se le decisioni che riguardano i cittadini sono giustificate, la nuova governance sarà capace di rigenerare circuiti democratici ${ }^{83}$.

Occorre anche ricordare che un'autorevole dottrina ritiene la governance disegnata su una organizzazione eterogenea ma fortemente interdipendente quale è l'Unione Europea come sperimentalista, e il necessario sperimentalismo può trasformare la diversità da ostacolo ad abbrivio del suo avanzamento. E all' incertezza strategica come condizione di possibilità che si attui la governance sperimentalista, si affianca, come ulteriore condizione, la distribuzione multipolare del potere, nell'ambito della quale nessun attore possa avere la capacità di imporre la sua soluzione preferita agli altri, senza tener conto della loro posizione ${ }^{84}$.

Una nuova sfida, dunque, per la comparazione giuridica e per imprimere una spinta orientativa alla vorticosa evoluzione del diritto, affinché una dottrina non segmentata da barriere nazionali e linguistiche produca nuove forme e categorie giuridiche, rispondenti alle esigenze della società europea ${ }^{85}$. Inforcati, ancora una volta, gli occhiali dello storico, che si sovrappongono, infine, a quelli del giurista, ci piace ricordare come la lunga avventura dello ius commune, che ha attraversato nei secoli l'Europa, assurga a modello, lontano e insuperato, d'una scienza giuridica che fece della dialettica critica del dialogo l'unità di popoli diversi.

${ }^{81}$ H. W. Micklitz, «The visible Hand of European Regulatory Private Law. The Transformation of European Private Law from Autonomy to Functionalism in Competition and Regulation», Yearbook of European Law, n. 28 (2009), pp. 3-59, e ID., «Introduction- Social Justice and Access Justice in Private Law», in H. W. MickLitz (cur.), The Many Concepts of Social Justice in European Private Law, Northampton (MA), Edward Elgar, 2011, pp. 3-60.

${ }^{82}$ L. RafFini, La democrazia in mutamento. Dallo Stato-nazione all'Europa, Firenze, Firenze University Press, 2010, pp. 99-100.

${ }^{83}$ C. F. SABeL e J. Cohen, «Sovereignty and Solidarity. EU and Us», in K. H. Ladeur (cur.), Public Governance in the Age of Globalization, Aldershot, Ashgate, 2004, pp. 157-175.

${ }^{84}$ C. F. SABEL e J. COHEN, «Governance sperimentalista», in C. F. SABEL (cur.), Esperimenti di nuova democrazia. Tra globalizzazione e localizzazione, Roma, Armando, 2013, p. 208.

${ }^{85}$ L. Antoniolul, «Lidentità delle scienze giuridiche in ordinamenti multilivello: il diritto comparato», in V. BARSOTTI (cur.), L'identità delle scienze giuridiche in ordinamenti multilivello. Quaderni del Dottorato Fiorentino in Scienze Giuridiche, Sant'Arcangelo di Romagna, Maggioli, 2014, pp. 1-27 e 23-24, in particolare p. 27. 
Ancora una volta, l'Europa dovrebbe reinventarse a sí misma in uno scenario nuovo, come quando, nel Medio Evo, mutamenti scatenati su ogni fronte possibile richiesero, de forma urgente e inmediata, un nuovo ordine giuridico $^{86}$.

Come è noto, non si costruisce il futuro senza conoscere il passato, senza sapere chi sono e da dove vengo prima di programmare dove vado. Un'analisi dell'unità culturale che era alla base dello ius commune rivela esattamente quello che manca ai nostri tempi, concentrati maggiormente sui fattori economici e politici piuttosto che sul substrato identificativo delle istituzioni europee. Per comprendere lo que somos —in modo irrevocabile, por mera decantación histórica ${ }^{87}$-, lo ius commune è una possibile chiave, in quanto mostra un modello di relazione tra ordinamenti giuridici plurali, con fonti plurali e plurali centri di costruzione normativa. Costituisce un chiaro precedente per fronteggiare la congerie plurinormativa e multilivello europea, in grado di offrire risposte agli attuali interrogativi giuridici, pur se nel mondo del diritto nulla è mai definitivo. Il tentativo, o meglio, l'aspirazione, dei giuristi rimane sempre di essere ordenadores del caos $^{88}$.

$\mathrm{Su}$ un altro fronte, poi, quello che oggi maggiormente ci condiziona, forse è giunto il momento di recuperare il pensiero del raffinato apologeta inglese della Rivoluzione Americana per provare a riflettere nuovamente sulle differenze che intercorrono tra una Costituzione ereditata e una Costituzione rivoluzionaria, assimilando a una Carta ereditata quella che regola uno Stato-nazione, e a una rivoluzionaria, nel senso di innovativa, la futura Costituzione europea. Dalle pagine di Burke sulla costruzione del nuovo ordine giuridico americano, ancorché politico, affiora l'intento di offrire una chiave di volta per estinguere il conflitto tra le colonie e la madrepatria, declinato mediante l'analisi delle vicende storiche, delle

${ }^{86}$ F. Martínez Martínez, «Ius commune, Utrumque ius: tiempos de Derecho único, tiempos de juristas», Glossae. European Journal of Legal History, n. 13 (2016), pp. 372-423, in particolare pp. 395 e 398. L'autore ricorda che il diritto comune fu l'opera collettiva de todos y dirigido a todos, orden compartido por toda la Europa occidental, grazie alla sua forza di espansione nel tempo e nello spazio e all'ampiezza dei suoi molteplici contenuti. Fu comune, e generale, «porque se edificó sobre presupuestos ligados a la naturaleza bumana, inmutables, no cambiantes y no dependientes de otras circunstancias y motivaciones más evanescentes» (p. 379).

${ }^{87}$ Questa Europa desnortada, mercantil, al margine della quale rimangono problemi enormi — come, ad esempio, quello dei rifugiati- che finiscono col disintegrarne l'unitarietà, non può essere compresa «sin Roma, sin Atenas y sin Jerusalén, sin el Derecho romano, sin la Filosofía griega y sin la Religión judeocristiana. No se trata de volver sobre esas huellas», si tratta piuttosto di comprendere il passato e di captarne l'essenza. Si veda ibid., pp. 383-384.

${ }^{88}$ Ibid., p. 385. 
componenti culturali e religiose, teso a evidenziare i principî comuni, piuttosto che le divergenze, tra il primigenio pianeta e il satellite che, con un proprio moto di rivoluzione, sfugge ormai la sua orbita. Come è noto, la secessione innesca un difficile processo che durerà per un lungo periodo in cui, a partire dalla Dichiarazione dei Diritti, si scontrano due posizioni diverse, l'una tendente a evitare che si crei un super-potere rispetto ai singoli Stati, l'altra, prevalente, che per non dissolvere la nuova entità che si è costituita, al contrario concretizza e rafforza il potere del governo federale. Mutatis mutandis, tale dibattito costituzionale appare di estremo interesse ancor oggi, in quanto muove dall'assoluta mancanza di una omogeneità delle posizioni politiche. La riflessione costituzionale in America produce la costruzione di un organismo articolato; alla Carta fondamentale, alcuni emendamenti verranno aggiunti successivamente: il punto d'equilibrio è raggiunto dal riconoscimento di un ampia libertà degli Stati federati almeno per quello che riguarda l'amministrazione interna; il Presidente eletto da tutti è un elemento di raccordo prevalentemente per quanto riguarda la politica estera, la guerra e la pace. Nessuna novità: il modello di Burke, pur nelle differenze, resta quello della madrepatria. Ma non può rifuggire alla realtà fattuale, quella che vede in atto un movimento, dapprima indipendentista, quindi fondativo di un nuovo ordinamento, diretto a creare una democrazia costituzionale e rappresentativa ${ }^{89}$. L'azione sopravanza la teoria dalla quale origina.

Nell'epoca globale, per richiamarci all'assunto iniziale di questo lavoro, relativo al recupero del senso delle parole, e, in particolare, dei lemmi della politica, ripensare il costituzionalismo, significa preliminarmente ridefinire il termine Costituzione, ripercorrendo le tappe del processo della sua risemantizzazione, che nel corso degli ultimi anni gli ha conferito una impressionante varietà di caratterizzazioni a scapito della tradizionale storia del concetto. Nell'ottica normativa che è all'origine del «paradigma del costituzionalismo moderno, inteso come scienza di fondazione e di limitazione del potere legittimo ${ }^{90}$ non si può non rilevare che il linguaggio del costituzionalismo sia tornato a godere di grande diffusione e influenza nell'ambito dell'intenso dibattito sul nuovo ordine giuridico e politico della società globale ${ }^{91}$.

${ }^{89}$ Si consenta il riferimento a R. AliBRANDI, Rivoluzione Sovranità Libertà..., op. cit., pp. 189-193.

${ }_{90}$ G. Azzariti, Il costituzionalismo moderno può sopravvivere?, Roma-Bari, Laterza, 2013 , pp. 26 y ss.

${ }_{91}$ M. Kumm, «The Cosmopolitan Turn in Constitutionalism: On the Relationship 
Alla base di questo fenomeno, c'è sicuramente il senso di sperdimento prodotto, anche a livello teorico, dagli intensi e tumultuosi processi giuridico-politici che hanno fatto seguito alla crisi dell'ordine bipolare del mondo. Quando si è costretti a muoversi in spazi non familiari, diviene infatti naturale tentare di riorientarsi utilizzando le mappe categoriali che già si posseggono, e «lavorare con le analogie, estendere e adattare i concetti esistenti, sembra solitamente preferibile a creare idee e strutture partendo da zero, non solo a causa degli impliciti rischi, ma anche per i nostri limiti di immaginazione» ${ }^{92}$.

La dottrina giuridica contemporanea vive la necessità di uno sforzo di adeguamento delle consolidate e millenarie sue categorie, oltre che del proprio vocabolario, preambolo di una rifondazione di teoria e concetti che suppliscano ai vuoti d'un sistema unitario più presupposta, o sognata, che reale.

E la storia recente dimostra come l'Unione Europea, che non è riuscita a darsi una Costituzione, non sia mai stata un'unione politica, ma si sia piuttosto rivelata una mera costruzione giuridica, tuttavia in grado di incidere sulle legislazioni nazionali e di imporre scelte economiche al di fuori di ogni procedura democratica. La sua vera natura si è manifestata al momento della crisi economica che ha colpito in modo diverso i vari Stati membri, evidenziando impietosamente le gerarchie esistenti; sotto il profilo squisitamente economico, si deve rilevare che l'irrompere della débâcle dei debiti sovrani, proprio a causa dell'estrema impreparazione istituzionale dell'Unione, ha rischiato di far fallire i Paesi con debiti eccessivi, come pure di far naufragare lo stesso progetto di integrazione politica dell'UE ${ }^{93}$.

Riguardo all'Italia, gli obblighi derivanti dalla sua partecipazione alla Nato e alla UE, non solo ne hanno eroso la sovranità (ricordiamo che le funzioni di battere moneta e decidere sulla guerra costituiscono i pilastri

between Constitutionalism in and beyond the State», in J. F. Dunoff e J. P. Trachtman (curr.), Ruling the World? Constitutionalism, International Law and Global Governance, Cambridge, Cambridge University Press, 2009, p. 258.

${ }^{2}$ N. Krisch, Beyond Constitutionalism. The Pluralist Structure of Postnational Law, Oxford, Oxford University Press, 2010, p. 27 (traduzione nostra).

${ }_{93} \mathrm{La}$ crisi economica globale ha causato enormi mutamenti negli scenari economici internazionali e ha prodotto effetti nefasti su quella che poteva considerarsi una costituzione economica europea con ripercussioni anche nella sfera del welfare, interdipendente dal mercato. Le sempre più marcate disparità economico-sociali e l'incapacità dell'Unione di comprenderle e ricomporle minano in radice la legittimità dell'Unione e sono, altresì, la manifestazione di un latente deficit di razionalità della costruzione. Si veda F. Losurdo, Lo Stato sociale condizionato: Stabilità e crescita nell'ordinamento, Torino, Giappichelli, 2016, pp. 3-8. 
su cui si fonda la sovranità dello Stato moderno), ma hanno stretto le istituzioni in una rete di condizionamenti e di divieti che segnano gli spazi politici entro cui possono muoversi governo, parlamento e persino la presidenza della Repubblica. «A questo si è giunti sia per le scelte di fondo attuate da governo e parlamento negli anni del dopoguerra, sia per decisioni assunte successivamente, nella Prima e nella Seconda repubblica, da parte di maggioranze anche di diverso colore, scelte legate tra loro nel tempo, motivate spesso ideologicamente, spesso non valutate nelle loro implicazioni e conseguenze» ${ }^{94}$.

E mentre non nasce una Costituzione europea, della quale non è poi detto che - specie a livello dei singoli cittadini- si avverta la mancanza, si è recentemente propugnata una sostanziale riforma di quella Costituzione che ancor oggi mantiene una forte carica di utopia positiva, eretta a baluardo contro i predatori del potere. E che, pur non essendo immutabile, «impone di mantenere integri i princìpi fondamentali, che non sono confinati nella Parte prima, ma trovano nella Parte seconda gli strumenti istituzionali atti a renderli effettivi». Inoltre, giusto per richiamare una delle istanze fondanti del multilevel constitutionalism (nel cui quadro le regioni sono gli emerging political actors ${ }^{95}$ ovvero la partecipazione alla governance anche dei livelli subnazionali, appare evidente come non vi possa essere una autentica autonomia regionale se, a livello di Stato centrale «non esisterà un'istanza democratica di contrappeso territoriale all'indirizzo politico nazionale. [...] Il sistema continuerebbe a tendere verso il centralismo e l'autonomia regionale sarebbe di nuovo affidata alle "cure" della Corte costituzionale, che necessariamente interviene ex post, con strumenti inadeguati a modificare e modulare gli indirizzi politici nazionale e locali. Il processo politico continuerebbe a rifluire in quello giurisdizionale, con tutte le rigidità e l'episodicità che ciò comporta» ${ }^{96}$. E proprio adesso che la crisi della sovranità postula la ricerca di un nuovo equilibrio costituzionale, anche confrontandosi all'esperienza del costituzionalismo europeo, il mantenimento di tale equilibrio è più che mai essenziale ${ }^{97}$.

94 G. SCARPARI, «Sovranità limitata», op. cit., p. 13.

95 J. Mathias e A. Stevens, «Regions and regional Politics in Europe», in R. Sakwa e A. Stevens, Contemporary Europe, New York, Palgrave Macmillan, 2012, pp. 197-216, in particolare p. 201.

96 G. SiLvestri, «Rileggendo, sessant'anni dopo, il saggio di Temistocle Martines sull'autonomia politica delle regioni in Italia», Diritti regionali. Rivista di diritto delle autonomie territoriali, n. 3 (2016), pp. 1-8, in particolare pp. 7-8.

97 G. Silvestri, «Costituzionalismo e crisi dello Stato-nazione...», op. cit., p. 914. 
La linea di confine dello Stato di diritto si assottiglia in modo irrecuperabile, se è possibile ipotizzare che una riforma costituzionale stravolga forma di Stato e forma di governo; peraltro, laddove si rilevi che davvero la maggioranza dei consociati avverta la necessità di riforme tanto importanti, sarebbe auspicabile che, piuttosto che interventi di un governo designato e non eletto - sempre più somiglianti a colpi di mano e fondati su maggioranze risicate, in un parlamento che sta fagocitando se stesso-, si indícano elezioni che, preferibilmente in base a un sistema proporzionale puro, legittimino una nuova assemblea costituente ${ }^{98}$.

E poi, non è dimostrato che la maggioranza — anche quando affermi (ad esempio con una vittoria elettorale) il proprio assunto- abbia sempre ragione. Una saggia moderazione induce a considerare nefaste le vittorie totali di una parte politica sull'altra. Nel 1945 Luigi Einaudi, scrisse un magistrale saggio che chiariva il significato dello spirito di moderazione e descriveva come «atmosfera del compromesso e dell'accessione» il clima di unanimità di intenti e la ricerca di accordo non risultanti dal «do ut des fra interessi opposti» ma dalla discussione critica fra maggioranza e minoranza attraverso cui le parti in lotta riconoscono i propri limiti. In sostanza, lodava la buona volontà dei rappresentanti politici del popolo, la loro capacità di giungere al «superamento degli opposti in una unità superiore». Con questi presupposti, la legge può essere «osser-

98 In Italia, il 4 dicembre 2016, i cittadini si sono pronunciati contro la sostanziale riforma della Costituzione repubblicana. La sovranità popolare, esercitata mediante lo strumento referendario, con la vittoria del «No», si è chiaramente espressa indicando che la via per l'eventuale revisione del testo costituzionale, è da percorrere secondo procedure formali e con modalità politicamente corrette. Senza voler entrare nel merito, anche se è vero che, in passato, non sono mancate iniziative governative approdate in leggi di revisione della Costituzione, che non hanno sollevato obiezioni perché di contenuto decisamente minore rispetto all'ampiezza del progetto che è stato oggetto del referendum, è di norma inopportuno che il governo proponga e sostenga disegni di legge di revisione della Costituzione, per ragioni inerenti all'essenza della libertà del voto e, quindi, all'essenza stessa della democrazia, in quanto i parlamentari, legati dal rapporto di fiducia, sono indotti ad appoggiare le iniziative governative anche quando non le condividano. Inoltre, il sostegno governativo all'approvazione referendaria del progetto di riforma altera il confronto tra le parti sociali (si veda G. D'ELIA, A. RENTERÍA DíAz e M. P. Viviani, «Referendum costituzionale: le ragioni del NO», Altalex, disponibile in http://www.altalex.com/documents/news/2016/11/18/referendum-costituzionale -le-ragioni-del-no). La cospicua affluenza al voto (ha votato il 70 por 100 degli aventi diritto) è da considerarsi un fatto politico di grande rilievo, in quanto, attuando un'inversione di tendenza rispetto all'astensionismo che ha connotato le ultime consultazioni, ha dimostrato come l'elettorato avesse chiara l'importanza della materia referendaria e, altresì, quanto profondamente il popolo italiano avverta che la Carta costituzionale, estrema garanzia della democrazia, della convivenza civile e della tutela dei diritti fondamentali, sia un valore da custodire. 
vata da tutti, è legge attiva e fruttuosa perché è frutto del compromesso fra gli opposti [...]. Soltanto allora il popolo dice: questa è la legge. E ad essa ubbidisce» ${ }^{99}$.

Oggi, anche chi non lo è mai stato si ritrova ad essere ideologicamente conservatore, laddove questa voce risalente, conservare, rinnova nell'agone politico il suo primario significato: difendere le libertà civili e politiche conquistate in un recente passato o, in tre parole, conservare la Costituzione; rivaluta, al contempo, un altro concetto antico, quello della rappresentanza politica. E rilegge - e rimedita - un'altra pagina nella quale lo statista piemontese pone il quesito finale: quid agendum? Bisogna assistere inerti alla morte della libertà? ${ }^{100}$

99 L. EINAUDI, «Major pars et sanior pars, ossia della tolleranza e dell'adesione politica (1945)», in E. Rossi (cur.), Il buongoverno, Bari, Laterza, 1954, pp. 111-112.

${ }^{100}$ L. EINAUDI, «La forza solo contro la forza», in Risorgimento liberale, 9 de marzo de 1945, p. 3.

Foro, Nueva época, vol. 19, núm. 2 (2016): 57-95 
